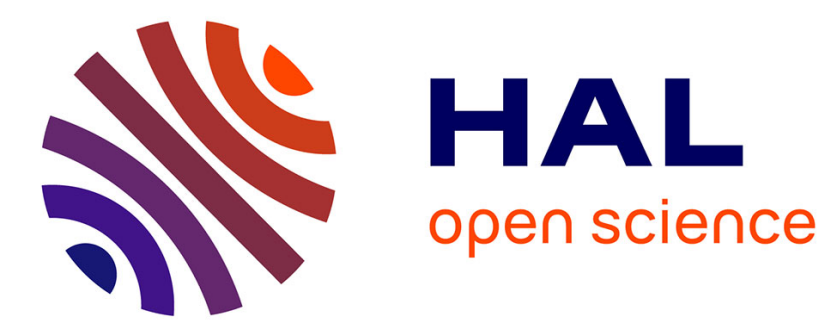

\title{
Modal criteria for optimizing the acoustic behaviour of a coupled fluid-structure system based on a systemic approach
}

Sébastien Besset, Louis Jezequel

\section{> To cite this version:}

Sébastien Besset, Louis Jezequel. Modal criteria for optimizing the acoustic behaviour of a coupled fluid-structure system based on a systemic approach. International Journal for Numerical Methods in Engineering, 2008, 73 (10), pp.1347-1373. 10.1002/nme.2125 . hal-00625130

\section{HAL Id: hal-00625130 \\ https://hal.science/hal-00625130}

Submitted on 26 Sep 2011

HAL is a multi-disciplinary open access archive for the deposit and dissemination of scientific research documents, whether they are published or not. The documents may come from teaching and research institutions in France or abroad, or from public or private research centers.
L'archive ouverte pluridisciplinaire HAL, est destinée au dépôt et à la diffusion de documents scientifiques de niveau recherche, publiés ou non, émanant des établissements d'enseignement et de recherche français ou étrangers, des laboratoires publics ou privés. 


\title{
Modal Criteria for Optimizing the Acoustic Behaviour of a Coupled Fluid-Structure System Based on a Systemic Approach
}

\author{
S. Besset, L. Jézéquel \\ LTDS, Équipe D2S, UMR CNRS 5513 \\ École Centrale de Lyon \\ 36 av. Guy de Collongue \\ 69134 Ecully cedex, France
}

International Journal for Numerical Methods in Engineering 73(10), 1347-1373, 2008

\begin{abstract}
This paper proposes modal criteria to represent various noise sources within a complex structure, such as an automobile. By optimizing a complex system using criteria linked to modal mass and stiffness matrices, different modes of noise propagation can be investigated separately. Several criteria are thus suggested, each related to a vibrational propagation path. Since the system is studied using modal analysis, criteria can be found based on modes associated with the structure's hollow parts, plates, and cavities. These different criteria are analyzed based on the assumption of a complex vibroacoustic system. It is shown that by analyzing such criteria, one can determine which part of the structure needs to be optimized. The optimization of such a system could constitute a research topic in its own right, and is beyond the scope of the present paper.
\end{abstract}

keywords: Modal analysis; Optimization; Vibroacoustic coupling

\section{Introduction}

The use of a modal approach to describe a structure in order to optimize its dynamic behaviour has multiple advantages. Once modal matrices have been computed, optimization criteria can readily be defined. The dynamic amplfication phenomena and dynamic coupling between substructures can then be described using just a small number of degrees of freedom. Furthermore, it is possible to link the criteria to the modal parameters used in the systemic procedure. The criteria proposed in this paper are derived from those developed by P. Lemerle [1], using Craig \& Bampton's method to describe the dynamic behaviour of a structure.

The modal analysis technique described here is based on the "double modal synthesis" proposed by Jézéquel [2, 3]. Complex structures often include hollow parts 
and stiffeners, which must be analyzed very accurately to obtain satisfactory results. In this paper, the term "hollow parts" denotes the formed steel and stiffeners making up the skeleton of a structure. In complex structures such as automobiles, stiffeners and formed steel parts which make up the skeleton of the structure are those most responsible for the behaviour of the structure as a whole. To analyze these elements, a method will be used, which was proposed in [4, 5]. We will study the acoustic parts of the coupled system using acoustic modes based on a "triple modal synthesis method". This method resorts to a coupling formulation which has been previously investigated [6. 7]. An example of modal analysis of a coupled system can be found in [8].

Modal analysis of the structure will lead to modal mass and stiffness matrices, which are then used to obtain effective modal parameters, which in turn lead to criteria allowing the structure to be optimized. These criteria will depend on the pressure values at points located in the acoustic parts of the system - inside a car for example - as a function of an excitation point located on a hollow part of the structure - e.g. a spar near the car engine. The criteria proposed here allow various vibrational propagation paths to be considered. It is thus possible to investigate separately the different noise sources within the structure.

The method proposed in this paper may be used for any vibroacoustic system. In fact, the ultimate goal of this approach is to define modal criteria which allow the vibroacoustic system to be optimized. These are related to the coupling terms between the systems different substructures, and are expressed as functions of the terms contained in the modal matrices. Thus, the number of criteria depends on the number of substructures within the vibroacoustic system. Our method is described for the case of a specific system, but can readily be adapted to other vibroacoustic systems.

The structure considered in this paper is a complex one, comprised of acoustic cavities, hollow parts and plates. The geometry of this structure is similar to that of a car. Two cavities will be considered. Thus, the method proposed here can be used to study the different paths through which vibrations propagate and generate noise within the car. Paths can exist through the hollow parts of the structure, through the plates which bound the cavity, and through plates which partition the two cavities. Such a structure has already been used in vacuo in [5].

\section{Analysis of the vibroacoustic system}

The structure considered here is a complex structure including hollow parts and plates. It is built using formed steels which make up its skeleton, as shown in figure 1. Plates are fixed to this skeleton, and form two cavities inside the structure, as shown. The structure's geometry is similar to that of a car, in order to show that the proposed methods can be applied in an industrial context.

[Figure 1 about here.]

\subsection{The vibroacoustic problem}

Figure 1 shows the geometry of the vibroacoustic system under consideration. The approach used in this paper to study the vibroacoustic behaviour is a $(\mathbf{u}, p)$ formulation: 


$$
\begin{array}{cl}
\sigma_{i j, j}(\mathbf{u})+\omega^{2} \rho_{s} \mathbf{u}_{i}=0 & \text { over } \Omega_{s} \\
\Delta p+\frac{\omega^{2}}{c^{2}} p=0 & \text { over } \Omega_{f} \\
\sigma_{i j}(\mathbf{u}) \mathbf{n}_{j}^{s}=\mathbf{f}_{i} & \text { over } \partial \Omega_{s} \backslash \Sigma \\
\sigma_{i j}(\mathbf{u}) \mathbf{n}_{j}^{s}=-p \mathbf{n}_{i}^{s}=p \mathbf{n}_{i}^{f} & \text { over } \Sigma \\
\frac{\partial p}{\partial \mathbf{n}^{f}}=\omega^{2} \rho_{f} \mathbf{u} \cdot \mathbf{n}^{f} & \text { over } \Sigma \\
\mathbf{A u}=0 & \text { over } \Sigma_{l}
\end{array}
$$

where $\Omega_{s}$ is the structural part, $\Omega_{f}$ is the acoustic part, and $\Sigma$ is the boundary between the structural and acoustic parts. $\Sigma_{l}$ is that part of the structure which is submitted to boundary conditions. $\mathbf{A}$ is an operator that allows these boundary conditions to be applied. The notations used in the equations describing the system are given below:

- $\sigma_{i j}$ is the stress tensor of the structure;

- $\omega$ is the angular frequency of the system;

- $\rho_{s}$ is the density of the structure;

- $\mathrm{f}$ represents the forces applied to the structure;

- $\mathbf{u}$ is the displacement of the structure;

- $p$ is the pressure field of the acoustic part.

The dissipation model (modal damping) will be introduced through the modal synthesis of the system in section 2.3 The next section addresses the discretization of the equations when several structural parts (plates and hollow parts) and two acoustic cavities are assumed. This model represents a car which is fixed to four points corresponding to the wheels; therefore, no rigid body modes are involved in the case studied here. Moreover, no internal acoustic source is assumed to be present.

\subsection{Finite element analysis of the system}

The behaviour of the vibroacoustic system studied in this paper can be approximated by the finite element model according to the following equilibrium motion equation: 


$$
\begin{aligned}
& \left(-\omega^{2}\left[\begin{array}{ccc|cc}
\mathbf{M}_{H H} & \mathbf{M}_{H P_{1}} & \mathbf{M}_{H P_{2}} & & \\
\mathbf{M}_{P_{1} H} & \mathbf{M}_{P_{1} P_{1}} & \mathbf{M}_{P_{1} P_{2}} & & \mathbf{0} \\
\mathbf{M}_{P_{2} H} & \mathbf{M}_{P_{2} P_{1}} & \mathbf{M}_{P_{2} P_{2}} & & \\
\hline & \mathcal{C}_{F} & & \mathbf{M}_{F_{1} F_{1}} & \mathbf{M}_{F_{1} F_{2}} \\
& & & \mathbf{M}_{F_{2} F_{1}} & \mathbf{M}_{F_{2} F_{2}}
\end{array}\right]\right. \\
& \left.+\left[\begin{array}{ccc|cc}
\mathbf{K}_{H H} & \mathbf{K}_{H P_{1}} & \mathbf{K}_{H P_{2}} & \\
\mathbf{K}_{P_{1} H} & \mathbf{K}_{P_{1} P_{1}} & \mathbf{K}_{P_{1} P_{2}} & -\mathcal{C}_{F}^{T} \\
\mathbf{K}_{P_{2} H} & \mathbf{K}_{P_{2} P_{1}} & \mathbf{K}_{P_{2} P_{2}} & \\
\hline & \mathbf{0} & & \mathbf{K}_{F_{1} F_{1}} & \mathbf{K}_{F_{1} F_{2}} \\
& \mathbf{0} & & \mathbf{K}_{F_{2} F_{1}} & \mathbf{K}_{F_{2} F_{2}}
\end{array}\right]\right)\left\{\begin{array}{c}
\mathbf{u}_{H} \\
\mathbf{u}_{P_{1}} \\
\mathbf{u}_{P_{2}} \\
\mathbf{p}_{F_{1}} \\
\mathbf{p}_{F_{2}}
\end{array}\right\}=\left\{\begin{array}{c}
\mathbf{f}_{H} \\
\mathbf{f}_{P_{1}} \\
\mathbf{f}_{P_{2}} \\
\mathbf{0} \\
\mathbf{0}
\end{array}\right\}
\end{aligned}
$$

The behaviour of the plates, the hollow parts and the cavities is included within this equation. Degrees of freedom denoted $\mathbf{u}_{H}$ correspond to the hollow parts; degrees of freedom denoted $\mathbf{u}_{P_{1}}$ correspond to the plates - with the exclusion of plates denoted $\mathcal{P}$ in figure 1-; degrees of freedom denoted $\mathbf{u}_{P_{2}}$ correspond to the plate denoted $\mathcal{P}$ in figure 1 Finally, degrees of freedom denoted $\mathbf{p}_{F_{1}}$ - respectively $\mathbf{p}_{F_{2}}$ - correspond to the pressure in the first cavity denoted $\mathcal{F}_{1}$ in figure 1- respectively the second cavity denoted $\mathcal{F}_{2}$ in figure 1

Thus, equation 7 is a classical interior non-symetric vibroacoustic equation. The degrees of freedom are displacements for the structural part, and pressure for the acoustic part.

The vibroacoustic coupling appears in matrice $\mathcal{C}_{F}$ defined as follows:

$$
\mathcal{C}_{F}=\left[\begin{array}{lll}
\mathbf{M}_{F_{1} H} & \mathbf{M}_{F_{1} P_{1}} & \mathbf{M}_{F_{1} P_{2}} \\
\mathbf{M}_{F_{2} H} & \mathbf{M}_{F_{2} P_{1}} & \mathbf{M}_{F_{2} P_{2}}
\end{array}\right]=-\left[\begin{array}{ll}
\mathbf{K}_{P_{2} F_{1}} & \mathbf{K}_{P_{2} F_{2}} \\
\mathbf{K}_{F_{1} F_{1}} & \mathbf{K}_{F_{1} F_{2}} \\
\mathbf{K}_{F_{2} F_{1}} & \mathbf{K}_{F_{2} F_{2}}
\end{array}\right]^{T}
$$

\subsection{Modal analysis of the system}

The acoustic part of the system is analyzed with a modal synthesis method using cavity modes. Hollow parts of the structure are analyzed using a model already proposed in [4]. These elements comprise the skeleton of the structure. This modeling leads to modal matrices which can be assembled in the same way as finite elements matrices. The main feature of this approach is that it produces matrices which have only generalized degrees of freedom. Nodal degrees of freedom may remain, in order to assemble the hollow parts to other structures, but boundaries between the substructures of the hollow part have only generalized degrees of freedom. Assembly of these substructures is made possible by the way in which the modes, used for the modal analysis of the substructures, are chosen. Plates are assembled to the structure's skeleton according to the nodal degrees of freedom remaining from the modal analysis of the hollow parts in the structure. The complete structure is then analyzed according to the "double modal synthesis" method proposed by Jézéquel [2, 3]. This method uses "branch modes" to describe the behaviour of the boundaries between substructures. In this paper, these 
"branch modes" will be used to describe the behaviour of the structure's skeleton. The use of generalized degrees of freedom allows the size of the problem to be reduced.

The degrees of freedom of a hollow part are comprised of generalized degrees of freedom resulting from the modal analysis of the substructures which make up the skeleton, denoted $\mathbf{q}_{H c}$, generalized degrees of freedom resulting from the double modal synthesis, which are denoted $\mathbf{q}_{H b}$, and excitation points, which are nodal degrees of freedom, denoted $\mathbf{u}_{E}$. We denote $\mathbf{q}_{H}=\left\{\begin{array}{c}\mathbf{u}_{E} \\ \mathbf{q}_{H c} \\ \mathbf{q}_{H b}\end{array}\right\}$.

Generalized degrees of freedom are linked to nodal degrees of freedom through the following equations:

$$
\left\{\begin{array}{l}
\mathbf{u}_{H c}=\boldsymbol{\Phi}_{H c} \mathbf{q}_{H c}+\boldsymbol{\Psi}_{H c} \mathbf{u}_{H b}+\boldsymbol{\Psi}_{H e} \mathbf{u}_{E} \\
\mathbf{u}_{H b}=\boldsymbol{\Phi}_{H b} \mathbf{q}_{H b} \\
\mathbf{u}_{P_{1}}=\boldsymbol{\Phi}_{P 1} \mathbf{q}_{P 1}+\boldsymbol{\Psi}_{P 1} \mathbf{u}_{H b}+\boldsymbol{\Psi}_{P 1 e} \mathbf{u}_{E} \\
\mathbf{u}_{P_{2}}=\boldsymbol{\Phi}_{P 2} \mathbf{q}_{P 2}+\boldsymbol{\Psi}_{P 2} \mathbf{u}_{H b}+\boldsymbol{\Psi}_{P 2 e} \mathbf{u}_{E} \\
\mathbf{p}_{F_{1}}=\boldsymbol{\Phi}_{F 1} \mathbf{q}_{F 1} \\
\mathbf{p}_{F_{2}}=\boldsymbol{\Phi}_{F 2} \mathbf{q}_{F 2}
\end{array}\right.
$$

Matrices $\boldsymbol{\Phi}_{F 1}$ and $\boldsymbol{\Phi}_{F 2}$ are the modal matrices of the acoustic modes of the system.

Matrices $\boldsymbol{\Phi}_{P 1}$ and $\boldsymbol{\Phi}_{P 2}$ are the modal matrices of the plates' fixed modes. $\boldsymbol{\Psi}_{P 1}$ and $\boldsymbol{\Psi}_{P 2}$ are the matrices of the plates' static modes, as in the case of the method used by Craig \& Bampton [9].

Matrices $\boldsymbol{\Phi}_{H c}$ and $\boldsymbol{\Psi}_{H c}$ are modal matrices resulting from the analysis of the hollow part described in [4] and [5]. Although this approach to the study of hollow parts has already been described in former papers, the reader will find a summary in the following.

Matrix $\boldsymbol{\Phi}_{H b}$ is the matrix of the "branch" modes of the structure [2, 3].

Excitation points, denoted $\mathbf{u}_{E}$ in the following, remain nodal degrees of freedom. Modal synthesis of the structure is not applied to these points.

The modal synthesis method used to study the hollow parts involves "section modes": The hollow part as a whole is split into several elements (substructures), as shown in figures 2 and 3. An element of a hollow part is studied with respect to the modes of the "left" and "right" sections. Elements are then assembled. This approach is used because it would be too costly, in terms of computing power, to study the structure's hollow parts using a conventional modal synthesis method.

[Figure 2 about here.]

[Figure 3 about here.]

The equation of motion thus becomes: 


$$
\begin{aligned}
& \left(\begin{array}{ccccccc}
\overline{\mathbf{M}}_{E E} & \overline{\mathbf{M}}_{E H c} & \mathbf{0} & \overline{\mathbf{M}}_{E P 1} & \overline{\mathbf{M}}_{E P 2} & \mathbf{0} & \mathbf{0} \\
\overline{\mathbf{M}}_{H c E} & \mathbf{m}_{H c} & \overline{\mathbf{M}}_{H c H b} & \mathbf{0} & \mathbf{0} & \mathbf{0} & \mathbf{0} \\
\mathbf{0} & \overline{\mathbf{M}}_{H b H c} & \mathbf{m}_{H b} & \overline{\mathbf{M}}_{H b P 1} & \overline{\mathbf{M}}_{H b P 2} & \mathbf{0} & \mathbf{0} \\
\overline{\mathbf{M}}_{P 1 E} & \mathbf{0} & \overline{\mathbf{M}}_{P 1 H b} & \mathbf{m}_{P 1} & \mathbf{0} & \mathbf{0} & \mathbf{0} \\
\overline{\mathbf{M}}_{P 2 E} & \mathbf{0} & \overline{\mathbf{M}}_{P 2 H b} & \mathbf{0} & \mathbf{m}_{P 2} & \mathbf{0} & \mathbf{0} \\
\overline{\mathbf{M}}_{F 1 E} & \mathbf{0} & \overline{\mathbf{M}}_{F 1 H b} & \overline{\mathbf{M}}_{F 1 P 1} & \overline{\mathbf{M}}_{F 1 P 2} & \mathbf{m}_{F 1} & \mathbf{0} \\
\overline{\mathbf{M}}_{F 2 E} & \mathbf{0} & \overline{\mathbf{M}}_{F 2 H b} & \overline{\mathbf{M}}_{F 2 P 1} & \overline{\mathbf{M}}_{F 2 P 2} & \mathbf{0} & \mathbf{m}_{F 2}
\end{array}\right] \\
& +i \omega\left[\begin{array}{ccccccc}
\mathbf{0} & \mathbf{0} & \mathbf{0} & \mathbf{0} & \mathbf{0} & \mathbf{0} & \mathbf{0} \\
\mathbf{0} & \mathbf{c}_{H c} & \mathbf{0} & \mathbf{0} & \mathbf{0} & \mathbf{0} & \mathbf{0} \\
\mathbf{0} & \mathbf{0} & \mathbf{c}_{H b} & \mathbf{0} & \mathbf{0} & \mathbf{0} & \mathbf{0} \\
\mathbf{0} & \mathbf{0} & \mathbf{0} & \mathbf{c}_{P 1} & \mathbf{0} & \mathbf{0} & \mathbf{0} \\
\mathbf{0} & \mathbf{0} & \mathbf{0} & \mathbf{0} & \mathbf{c}_{P 2} & \mathbf{0} & \mathbf{0} \\
\mathbf{0} & \mathbf{0} & \mathbf{0} & \mathbf{0} & \mathbf{0} & \mathbf{c}_{F 1} & \mathbf{0} \\
\mathbf{0} & \mathbf{0} & \mathbf{0} & \mathbf{0} & \mathbf{0} & \mathbf{0} & \mathbf{c}_{F 2}
\end{array}\right] \\
& \left.+\left[\begin{array}{ccccccc}
\overline{\mathbf{K}}_{E E} & \mathbf{0} & \mathbf{0} & \mathbf{0} & \mathbf{0} & \overline{\mathbf{K}}_{E F 1} & \overline{\mathbf{K}}_{E F 2} \\
\mathbf{0} & \mathbf{k}_{H c} & \mathbf{0} & \mathbf{0} & \mathbf{0} & \overline{\mathbf{K}}_{H c F 1} & \overline{\mathbf{K}}_{H c F 2} \\
\mathbf{0} & \mathbf{0} & \mathbf{k}_{H b} & \mathbf{0} & \mathbf{0} & \overline{\mathbf{K}}_{H b F 1} & \overline{\mathbf{K}}_{H b F 2} \\
\mathbf{0} & \mathbf{0} & \mathbf{0} & \mathbf{k}_{P 1} & \mathbf{0} & \overline{\mathbf{K}}_{P 1 F 1} & \overline{\mathbf{K}}_{P 1 F 2} \\
\mathbf{0} & \mathbf{0} & \mathbf{0} & \mathbf{0} & \mathbf{k}_{P 2} & \overline{\mathbf{K}}_{P 2 F 1} & \overline{\mathbf{K}}_{P 2 F 2} \\
\mathbf{0} & \mathbf{0} & \mathbf{0} & \mathbf{0} & \mathbf{0} & \mathbf{k}_{F 1} & \mathbf{0} \\
\mathbf{0} & \mathbf{0} & \mathbf{0} & \mathbf{0} & \mathbf{0} & \mathbf{0} & \mathbf{k}_{F 2}
\end{array}\right]\right)\left\{\begin{array}{c}
\mathbf{u}_{E} \\
\mathbf{q}_{H c} \\
\mathbf{q}_{H b} \\
\mathbf{q}_{P 1} \\
\mathbf{q}_{P 2} \\
\mathbf{q}_{F 1} \\
\mathbf{q}_{F 2}
\end{array}\right\}=\left\{\begin{array}{c}
\overline{\mathbf{f}}_{E} \\
\overline{\mathbf{f}}_{H c} \\
\overline{\mathbf{f}}_{H b} \\
\overline{\mathbf{f}}_{P 1} \\
\overline{\mathbf{f}}_{P 2} \\
\mathbf{0} \\
\mathbf{0}
\end{array}\right\}
\end{aligned}
$$

where matrices in lowercase characters are diagonal matrices. At this step of the modal synthesis, modal damping is introduced. Although it involves a small degree of approximation, the damping matrix is assumed to be diagonal. This property is called Basile's hypothesis is the french terminology. This has no impact on the results provided dissipation does not need to be analysed.

\section{Optimization criteria used in the paper}

As explained in the introduction, although the methodology described here can be adapted to any generalised vibroacoustic system, a specific example has been chosen to illustrate our methodology for deriving the optimization criteria (see figure1).

\subsection{Analysis of the motion equation}

The aim of this section is to express the pressure $\mathbf{p}_{F_{2}}$ in the second cavity as a function of the structural displacements. This expression will allow the various vibrational paths to be discriminated from one another. The problem must be studied using a systemic approach. Certain criteria related to the different vibrational paths will thus be defined.

Using the last line of equation 10 , the following expression is obtained: 


$$
\begin{aligned}
-\omega^{2}\left(\overline{\mathbf{M}}_{F 2 E}^{k} \mathbf{u}_{E}+\right. & \overline{\mathbf{M}}_{F 2 H b}^{k} \mathbf{q}_{H b} \\
& \left.+\overline{\mathbf{M}}_{F 2 P 1}^{k} \mathbf{q}_{P 1}+\overline{\mathbf{M}}_{F 2 P 2}^{k} \mathbf{q}_{P 2}+\mathbf{m}_{F 2}^{k} \mathbf{q}_{F 2}^{k}\right) \\
& +i \omega \mathbf{c}_{F 2}^{k} \mathbf{q}_{F 2}^{k}+\mathbf{k}_{F 2}^{k} \mathbf{q}_{F 2}^{k}=\mathbf{0}
\end{aligned}
$$

where $\overline{\mathbf{M}}_{F 2 E}^{k}, \overline{\mathbf{M}}_{F 2 H b}^{k}, \overline{\mathbf{M}}_{F 2 P 1}^{k}$ and $\overline{\mathbf{M}}_{F 2 P 2}^{k}$ are the $k^{\text {th }}$ lines of matrices $\overline{\mathbf{M}}_{F 2 E}$, $\overline{\mathbf{M}}_{F 2 H c}, \overline{\mathbf{M}}_{F 2 H b}, \overline{\mathbf{M}}_{F 2 P 1}$ and $\overline{\mathbf{M}}_{F 2 P 2} . \mathbf{q}_{F 2}^{k}$ is the $k^{\text {th }}$ component of vector $\mathbf{q}_{F 2}$.

Equation 11leads to:

$$
\mathbf{q}_{F 2}^{k}=\frac{\omega^{2}\left(\overline{\mathbf{M}}_{F 2 E}^{k} \mathbf{u}_{E}+\overline{\mathbf{M}}_{F 2 H b}^{k} \mathbf{q}_{H b}+\overline{\mathbf{M}}_{F 2 P 1}^{k} \mathbf{q}_{P 1}+\overline{\mathbf{M}}_{F 2 P 2}^{k} \mathbf{q}_{P 2}\right)}{-\omega^{2} \mathbf{m}_{F 2}^{k}+i \omega \mathbf{c}_{F 2}^{k}+\mathbf{k}_{F 2}^{k}}
$$

Let $\boldsymbol{\Phi}_{F 2}^{k}$ be the $k^{\text {th }}$ column of $\boldsymbol{\Phi}_{F 2}$. Equations 9 and 12 yield:

$$
\begin{aligned}
\mathbf{p}_{F_{2}}= & \sum_{k} \boldsymbol{\Phi}_{F 2}^{k} \mathbf{q}_{F 2}^{k} \\
= & \sum_{k}\left[\frac{\omega^{2} \boldsymbol{\Phi}_{F 2}^{k} \overline{\mathbf{M}}_{F 2 E}^{k}}{-\omega^{2} \mathbf{m}_{F 2}^{k}+i \omega \mathbf{c}_{F 2}^{k}+\mathbf{k}_{F 2}^{k}}\right] \mathbf{u}_{E} \\
& +\sum_{k}\left[\frac{\omega^{2} \boldsymbol{\Phi}_{F 2}^{k} \overline{\mathbf{M}}_{F 2 H b}^{k}}{-\omega^{2} \mathbf{m}_{F 2}^{k}+i \omega \mathbf{c}_{F 2}^{k}+\mathbf{k}_{F 2}^{k}}\right] \tilde{\boldsymbol{\Phi}}_{H b} \mathbf{u}_{H b} \\
& +\sum_{k}\left[\frac{\omega^{2} \boldsymbol{\Phi}_{F 2}^{k} \overline{\mathbf{M}}_{F 2 P 1}^{k}}{-\omega^{2} \mathbf{m}_{F 2}^{k}+i \omega \mathbf{c}_{F 2}^{k}+\mathbf{k}_{F 2}^{k}}\right]\left(\tilde{\boldsymbol{\Phi}}_{P 1}\left(\mathbf{u}_{P 1}-\mathbf{\Psi}_{P 1} \mathbf{u}_{H b}-\mathbf{\Psi}_{P 1 e} \mathbf{u}_{E}\right)\right) \\
& +\sum_{k}\left[\frac{\omega^{2} \boldsymbol{\Phi}_{F 2}^{k} \overline{\mathbf{M}}_{F 2 P 2}^{k}}{-\omega^{2} \mathbf{m}_{F 2}^{k}+i \omega \mathbf{c}_{F 2}^{k}+\mathbf{k}_{F 2}^{k}}\right]\left(\tilde{\boldsymbol{\Phi}}_{P 2}\left(\mathbf{u}_{P 2}-\mathbf{\Psi}_{P 2} \mathbf{u}_{H b}-\mathbf{\Psi}_{P 2 e} \mathbf{u}_{E} \delta 1\right) 3\right)
\end{aligned}
$$

The quantities with a "tilde" are left pseudo-inverse matrices. It is possible to define several pseudo-inverse matrices. In the case of singular systems, pseudo-inverse matrices allow solutions to be found [10]. In this paper, matrices are not square and the solution obtained is a least squares approximation. The reason for this is that modal synthesis does not involve fewer modes than the number of physical degrees of freedom of the system.

Equation 13 provides an approximation to the pressure field $\mathbf{p}_{F_{2}}$ within the second cavity as a function of structural displacements. A superposition of the substructural modes clearly appears in the sums $\sum_{k}($ ).

\subsection{Modal parameters}

Equation 13 allows modal parameters to be defined, which can be used to optimize the coupled system. These modal parameters correspond to each of the different vi- 
brational paths: a direct path, a path through the hollow parts of the structure, a path through the plates bounding the cavity, and a path through the plate located between the two cavities.

\subsubsection{Direct path}

The direct path is directly obtained through equation 13 It corresponds to an excitation point on a plate located next to cavity $\mathcal{F}_{2}$. It is recalled that the excitations considered here are displacement excitations.

The modal parameter corresponding to this direct path is denoted $\mathbf{G}_{E}(\omega)$ :

$$
\mathbf{G}_{E}(\omega)=\sum_{k} \frac{\left(\frac{\omega}{\omega_{k}}\right)^{2}}{1-\left(\frac{\omega}{\omega_{k}}\right)^{2}+2 i \xi_{k} \frac{\omega}{\omega_{k}}} \tilde{\mathbf{G}}_{E}^{k}
$$

where:

$$
\tilde{\mathbf{G}}_{E}^{k}=\frac{\boldsymbol{\Phi}_{F 2}^{k}\left(\overline{\mathbf{M}}_{F 2 E}^{k}-\overline{\mathbf{M}}_{F 2 P 1}^{k} \tilde{\boldsymbol{\Phi}}_{P 1} \boldsymbol{\Psi}_{P 1 e}-\overline{\mathbf{M}}_{F 2 P 2}^{k} \tilde{\boldsymbol{\Phi}}_{P 2} \boldsymbol{\Psi}_{P 2 e}\right)}{\mathbf{m}_{P 2}^{k}}
$$

with the notations $\mathbf{c}_{F 2}^{k}=2 \xi_{k} \sqrt{\mathbf{k}_{F 2}^{k} \mathbf{m}_{F 2}^{k}}$ and $\omega_{k}=\sqrt{\frac{\mathbf{k}_{F 2}^{k}}{\mathbf{m}_{F 2}^{k}}}$.

\subsubsection{Path through the hollow parts}

The path through the hollow parts is given by component $\mathbf{u}_{H b}$ of equation 13

$\mathbf{q}_{H b}^{k}$ is now expressed according to the third line of equation 10 .

$$
\begin{aligned}
& -\omega^{2}\left(\overline{\mathbf{M}}_{H b H c}^{k} \mathbf{q}_{H c}+\mathbf{m}_{H b}^{k} \mathbf{q}_{H b}^{k}+\overline{\mathbf{M}}_{H b P 1}^{k} \mathbf{q}_{P 1}+\overline{\mathbf{M}}_{H b P 2}^{k} \mathbf{q}_{P 2}\right) \\
& \quad+i \omega \mathbf{c}_{H b}^{k} \mathbf{q}_{H b}^{k}+\mathbf{k}_{H b}^{k} \mathbf{q}_{H b}^{k}+\overline{\mathbf{K}}_{H b F 1}^{k} \mathbf{q}_{F 1}+\overline{\mathbf{K}}_{H b F 2}^{k} \mathbf{q}_{F 2}=\overline{\mathbf{f}}_{H b}^{k}
\end{aligned}
$$

The modal parameter corresponding to the path through the hollow parts is denoted $\mathbf{G}_{H}(\omega)$. Using equations 16 and 11 it is possible to write:

$\mathbf{G}_{H}(\omega)=\left(\sum_{k} \frac{\left(\frac{\omega}{\omega_{1 k}}\right)^{2}}{1-\left(\frac{\omega}{\omega_{1 k}}\right)^{2}+2 i \xi_{1 k} \frac{\omega}{\omega_{1 k}}} \tilde{\mathbf{G}}_{H 1}^{k}\right)\left(\sum_{k} \frac{\left(\frac{\omega}{\omega_{2 k}}\right)^{2}}{1-\left(\frac{\omega}{\omega_{2 k}}\right)^{2}+2 i \xi_{2 k} \frac{\omega}{\omega_{2 k}}} \tilde{\mathbf{G}}_{H 2}^{k}\right)$

where:

$$
\begin{aligned}
\tilde{\mathbf{G}}_{H 1}^{k} & =\frac{\boldsymbol{\Phi}_{F 2}^{k} \overline{\mathbf{M}}_{F 2 H b}^{k} \tilde{\boldsymbol{\Phi}}_{H b}}{\mathbf{m}_{F 2}^{k}} \\
\tilde{\mathbf{G}}_{H 2}^{k} & =\frac{\boldsymbol{\Phi}_{H b}^{k}\left(\overline{\mathbf{M}}_{H b H c}^{k} \tilde{\boldsymbol{\Phi}}_{H c} \boldsymbol{\Psi}_{H e}-\overline{\mathbf{M}}_{H b P 1}^{k} \tilde{\boldsymbol{\Phi}}_{P 1} \boldsymbol{\Psi}_{P 1 e}-\overline{\mathbf{M}}_{H b P 2}^{k} \tilde{\boldsymbol{\Phi}}_{P 2} \boldsymbol{\Psi}_{P 2 e}\right)_{(19)}}{\mathbf{m}_{H b}^{k}}
\end{aligned}
$$


with the notations $\mathbf{c}_{F 2}^{k}=2 \xi_{1 k} \sqrt{\mathbf{k}_{F 2}^{k} \mathbf{m}_{F 2}^{k}}, \omega_{1 k}=\sqrt{\frac{\mathbf{k}_{F 2}^{k}}{\mathbf{m}_{F 2}^{k}}}$, and $\mathbf{c}_{H b}^{k}=2 \xi_{2 k} \sqrt{\mathbf{k}_{H b}^{k} \mathbf{m}_{H b}^{k}}$ and $\omega_{2 k}=\sqrt{\frac{\mathbf{k}_{H b}^{k}}{\mathbf{m}_{H b}^{k}}}$.

\subsubsection{Path through the plates}

The path through the plates is given by component $\mathbf{u}_{P 1}$ of equation 13

$\mathbf{u}_{P 1}$ is then written as a function of $\mathbf{u}_{E}$ according to the fourth line of equation 10 .

$$
\begin{aligned}
-\omega^{2}\left(\overline{\mathbf{M}}_{P 1 E}^{k} \mathbf{u}_{E}\right. & \left.+\overline{\mathbf{M}}_{P 1 H b}^{k} \mathbf{q}_{H b}+\mathbf{m}_{P 1}^{k} \mathbf{q}_{P 1}^{k}\right) \\
& +i \omega \mathbf{c}_{P 1}^{k} \mathbf{q}_{P 1}^{k}+\mathbf{k}_{P 1}^{k} \mathbf{q}_{P 1}^{k}+\overline{\mathbf{K}}_{P 1 F 1} \mathbf{q}_{F 1}+\overline{\mathbf{K}}_{P 1 F 2} \mathbf{q}_{F 2}=\overline{\mathbf{f}}_{P 1}
\end{aligned}
$$

The modal parameter corresponding to the path through the plates is denoted $\mathbf{G}_{P 1}(\omega)$. Using equations 20 and 11 it is possible to write:

$$
\begin{gathered}
\mathbf{G}_{P 1}^{1}(\omega)=\left(\sum_{k} \frac{\left(\frac{\omega}{\omega_{1 k}}\right)^{2}}{1-\left(\frac{\omega}{\omega_{1 k}}\right)^{2}+2 i \xi_{1 k} \frac{\omega}{\omega_{1 k}}} \tilde{\mathbf{G}}_{P 11}^{k}\right)\left(\sum_{k} \frac{\left(\frac{\omega}{\omega_{2 k}}\right)^{2}}{1-\left(\frac{\omega}{\omega_{2 k}}\right)^{2}+2 i \xi_{2 k} \frac{\omega}{\omega_{2 k}}} \tilde{\mathbf{G}}_{P 12}^{k}\right) \\
\mathbf{G}_{P 1}^{2}(\omega)=\left(\sum_{k} \frac{\left(\frac{\omega}{\omega_{1 k}}\right)^{2}}{1-\left(\frac{\omega}{\omega_{1 k}}\right)^{2}+2 i \xi_{1 k} \frac{\omega}{\omega_{1 k}}} \tilde{\mathbf{G}}_{P 11}^{k}\right) \\
\times\left(\sum_{k} \frac{\left(\frac{\omega}{\omega_{2 k}}\right)^{2}}{1-\left(\frac{\omega}{\omega_{2 k}}\right)^{2}+2 i \xi_{2 k} \frac{\omega}{\omega_{2 k}}} \tilde{\mathbf{G}}_{P 13}^{k}\right) \\
\times\left(\sum_{k} \frac{\left(\frac{\omega}{\omega_{3 k}}\right)^{2}}{1-\left(\frac{\omega}{\omega_{3 k}}\right)^{2}+2 i \xi_{3 k} \frac{\omega}{\omega_{3 k}}} \tilde{\mathbf{G}}_{P 14}^{k}\right)
\end{gathered}
$$

where:

$$
\begin{aligned}
\tilde{\mathbf{G}}_{P 11}^{k} & =\frac{\boldsymbol{\Phi}_{F 2}^{k} \overline{\mathbf{M}}_{F 2 P 1}^{k} \tilde{\boldsymbol{\Phi}}_{P 1}}{\mathbf{m}_{F 2}^{k}} \\
\tilde{\mathbf{G}}_{P 12}^{k} & =\frac{\boldsymbol{\Phi}_{P 1}^{k} \overline{\mathbf{M}}_{P 1 E}^{k}}{\mathbf{m}_{P 1}^{k}} \\
\tilde{\mathbf{G}}_{P 13}^{k} & =\frac{\boldsymbol{\Phi}_{P 1}^{k} \overline{\mathbf{M}}_{P 1 H b}^{k} \tilde{\boldsymbol{\Phi}}_{H b}}{\mathbf{m}_{P 1}^{k}} \\
\tilde{\mathbf{G}}_{P 14}^{k} & =\tilde{\mathbf{G}}_{H 2}^{k} \quad \text { (see equation 19) }
\end{aligned}
$$


with the notations $\mathbf{c}_{F 2}^{k}=2 \xi_{1 k} \sqrt{\mathbf{k}_{F 2}^{k} \mathbf{m}_{F 2}^{k}}$ and $\omega_{1 k}=\sqrt{\frac{\mathbf{k}_{F 2}^{k}}{\mathbf{m}_{F 2}^{k}}}, \mathbf{c}_{P 1}^{k}=2 \xi_{2 k} \sqrt{\mathbf{k}_{P 1}^{k} \mathbf{m}_{P 1}^{k}}$ and $\omega_{2 k}=\sqrt{\frac{\mathbf{k}_{P 1}^{k}}{\mathbf{m}_{P 1}^{k}}}, \mathbf{c}_{H b}^{k}=2 \xi_{3 k} \sqrt{\mathbf{k}_{H b}^{k} \mathbf{m}_{H b}^{k}}$ and $\omega_{3 k}=\sqrt{\frac{\mathbf{k}_{H b}^{k}}{\mathbf{m}_{H b}^{k}}}$.

\subsubsection{Path through the first cavity}

The path through the first cavity is related to the plate forming the boundary between the two cavities.

The modal parameter corresponding to this path can be written according to the fifth and sixth lines of equation 10 .

$$
\begin{array}{r}
-\omega^{2}\left(\overline{\mathbf{M}}_{P 2 E}^{k} \mathbf{u}_{E}+\overline{\mathbf{M}}_{P 2 H b}^{k} \mathbf{q}_{H b}+\mathbf{m}_{P 2}^{k} \mathbf{q}_{P 2}^{k}\right) \\
+i \omega \mathbf{c}_{P 2}^{k} \mathbf{q}_{P 2}^{k}+\mathbf{k}_{P 2}^{k} \mathbf{q}_{P 2}^{k}+\overline{\mathbf{K}}_{P 2 F 1}^{k} \mathbf{q}_{F 1}+\overline{\mathbf{K}}_{P 2 F 2}^{k} \mathbf{q}_{F 2}=\overline{\mathbf{f}}_{P 2} \\
-\omega^{2}\left(\overline{\mathbf{M}}_{F 1 E}^{k} \mathbf{u}_{E}+\overline{\mathbf{M}}_{F 1 H b}^{k} \mathbf{q}_{H b}+\overline{\mathbf{M}}_{F 1 P 1}^{k} \mathbf{q}_{P 1}+\overline{\mathbf{M}}_{F 1 P 2}^{k} \mathbf{q}_{P 2}^{k}+\mathbf{m}_{F 1}^{k} \mathbf{q}_{F 1}\right) \\
+i \omega \mathbf{c}_{F 1}^{k} \mathbf{q}_{F 1}^{k}+\mathbf{k}_{F 1}^{k} \mathbf{q}_{F 1}^{k}=\mathbf{0}
\end{array}
$$

The modal parameter corresponding to the path through the plates is denoted $\mathbf{G}_{P 2}(\omega)$. Using equations 27, 28 and 11 it is possible to write:

$$
\begin{aligned}
\mathbf{G}_{P 2}(\omega)=\left(\sum_{k} \frac{\left(\frac{\omega}{\omega_{1 k}}\right)^{2}}{1-\left(\frac{\omega}{\omega_{1 k}}\right)^{2}+2 i \xi_{1 k} \frac{\omega}{\omega_{1 k}}} \tilde{\mathbf{G}}_{P 21}^{k}\right) \\
\times\left(\sum_{k} \frac{1}{1-\left(\frac{\omega}{\omega_{2 k}}\right)^{2}+2 i \xi_{2 k} \frac{\omega}{\omega_{2 k}}} \tilde{\mathbf{G}}_{P 22}^{k}\right) \\
\times\left(\sum_{k} \frac{\left(\frac{\omega}{\omega_{3 k}}\right)^{2}}{1-\left(\frac{\omega}{\omega_{3 k}}\right)^{2}+2 i \xi_{3 k} \frac{\omega}{\omega_{3 k}}} \tilde{\mathbf{G}}_{P 23}^{k}\right)
\end{aligned}
$$

where:

$$
\begin{aligned}
\tilde{\mathbf{G}}_{P 21}^{k} & =\frac{\boldsymbol{\Phi}_{F 2}^{k} \overline{\mathbf{M}}_{F 2 P 2}^{k} \tilde{\mathbf{\Phi}}_{P 2}}{\mathbf{m}_{F 2}^{k}} \\
\tilde{\mathbf{G}}_{P 22}^{k} & =\frac{\boldsymbol{\Phi}_{P 2}^{k} \overline{\mathbf{K}}_{P 2 F 1}^{k} \tilde{\boldsymbol{\Phi}}_{F 1}}{\omega_{2 k}^{2} \mathbf{m}_{P 2}^{k}} \\
\tilde{\mathbf{G}}_{P 23}^{k} & =\frac{\boldsymbol{\Phi}_{F 1}^{k}\left(\overline{\mathbf{M}}_{F 1 E}^{k}-\overline{\mathbf{M}}_{F 1 P 1}^{k} \tilde{\boldsymbol{\Phi}}_{P 1} \boldsymbol{\Psi}_{P 1 e}-\overline{\mathbf{M}}_{F 1 P 2}^{k} \tilde{\boldsymbol{\Phi}}_{P 2} \boldsymbol{\Psi}_{P 2 e}\right)}{\mathbf{m}_{F 1}^{k}}
\end{aligned}
$$


with the notations $\mathbf{c}_{F 2}^{k}=2 \xi_{1 k} \sqrt{\mathbf{k}_{F 2}^{k} \mathbf{m}_{F 2}^{k}}$ and $\omega_{1 k}=\sqrt{\frac{\mathbf{k}_{F 2}^{k}}{\mathbf{m}_{F 2}^{k}}}, \mathbf{c}_{P 2}^{k}=2 \xi_{2 k} \sqrt{\mathbf{k}_{P 2}^{k} \mathbf{m}_{P 2}^{k}}$
and $\omega_{2 k}=\sqrt{\frac{\mathbf{k}_{P 2}^{k}}{\mathbf{m}_{P 2}^{k}}}, \mathbf{c}_{F 1}^{k}=2 \xi_{3 k} \sqrt{\mathbf{k}_{F 1}^{k} \mathbf{m}_{F 1}^{k}}$ and $\omega_{3 k}=\sqrt{\frac{\mathbf{k}_{F 1}^{k}}{\mathbf{m}_{F 1}^{k}}}$.

\subsection{Modal criteria}

The modal parameters defined in the previous sections lead to criteria that are defined as follows:

$$
\begin{aligned}
& C_{E}=\max _{k}\left|\frac{\left(\frac{\omega}{\omega_{k}}\right)^{2}}{1-\left(\frac{\omega}{\omega_{k}}\right)^{2}+2 i \xi_{k} \frac{\omega}{\omega_{k}}} \tilde{\mathbf{G}}_{E}^{k}\right| \\
& \left.C_{n}=\max _{k}\left|\frac{\left(\frac{\omega}{\omega_{1 k}}\right)^{2}}{1-\left(\frac{\omega}{\omega_{1 k}}\right)^{2}+2 i \xi_{1 k} \frac{\omega}{\omega_{1 k}}} \tilde{\mathbf{G}}_{n}^{k}\right| \quad \text { where } n \text { can be } H 1, P 11 \text { or } P \text { 2B } 4\right) \\
& C_{n}=\max _{k}\left|\frac{\left(\frac{\omega}{\omega_{2 k}}\right)^{2}}{1-\left(\frac{\omega}{\omega_{2 k}}\right)^{2}+2 i \xi_{2 k} \frac{\omega}{\omega_{2 k}}} \tilde{\mathbf{G}}_{n}^{k}\right| \\
& C_{P 14}=\max _{k}\left|\frac{\left(\frac{\omega}{\omega_{3 k}}\right)^{2}}{1-\left(\frac{\omega}{\omega_{3 k}}\right)^{2}+2 i \xi_{3 k} \frac{\omega}{\omega_{3 k}}} \tilde{\mathbf{G}}_{P 14}^{k}\right| \\
& C_{P 22}=\max _{k} \mid \frac{1}{1-\left(\frac{\omega}{\omega_{2 k}}\right)^{2}+2 i \xi_{2 k} \frac{\omega}{\omega_{2 k}}} \tilde{\mathbf{G}}_{P 22}^{k} \\
& C_{P 23}=\max _{k} \mid \frac{\left(\frac{\omega}{\omega_{3 k}}\right)^{2}}{1-\left(\frac{\omega}{\omega_{3 k}}\right)^{2}+2 i \xi_{3 k} \frac{\omega}{\omega_{3 k}}} \tilde{\mathbf{G}}_{P 23}^{k}
\end{aligned}
$$

Within the framework of an optimization problem, it is possible to use these criteria, for example, in order to optimize a structure's geometry. However, the criteria we propose do not allow derivation with respect to any one of the parameters of the structure (geometry of the hollow parts, thickness of the plates...), although many optimization methods require derivating these criteria. For that reason other criteria, related to the original ones, are defined in the next section.

\subsection{Derivable criteria}

As mentioned below, criteria $C_{n}$ (where $n$ can be $E, H 1, H 2, P 11, P 12, P 13, P 14$, $P 21, P 22$ or $P 23$ ) cannot be derived with respect to any parameter, although this 
would be useful in most optimization problems. To remedy this problem, we introduce criteria $\tilde{C}_{n}$, defined as follows:

$$
\tilde{C}_{n}=\frac{1}{4} \log \sum_{k}\left|\lambda(\omega, k) \tilde{\mathbf{G}}_{n}^{k}\right|^{4}
$$

where $\lambda(\omega)$ is a coefficient depending on $\omega$. This is the case, for example, of $\tilde{\mathbf{G}}_{E}^{k}$, $\lambda(\omega)=\frac{\left(\frac{\omega}{\omega_{k}}\right)^{2}}{1-\left(\frac{\omega}{\omega_{k}}\right)^{2}+2 i \xi_{k} \frac{\omega}{\omega_{k}}}$.

It can be shown that these criteria are very similar to the first ones, and have almost the same minima and maxima. To prove this, let us consider the following function $f_{p}$ :

$$
f_{p}: \quad x \longrightarrow \sqrt[p]{a_{1}(x)^{p}+a_{2}(x)^{p}+\cdots+a_{n}(x)^{p}}
$$

where $a_{q}(x)>0 \quad \forall q \leq n \forall x$. It is possible to write:

$$
\lim _{p \rightarrow+\infty} f_{p}(x)=\max \left[a_{1}(x), a_{2}(x), \ldots, a_{n}(x)\right]
$$

Specifically, since $a_{q}(x)>0 \quad \forall q \leq n \forall x$ :

$$
\begin{gathered}
\sqrt[p]{\left(\max \left[a_{1}(x), a_{2}(x), \ldots, a_{n}(x)\right]\right)^{p}}<f_{p}(x)<\sqrt[p]{n\left(\max \left[a_{1}(x), a_{2}(x), \ldots, a_{n}(x)\right]\right)^{p}} \\
\forall p \in \mathbb{N} .
\end{gathered}
$$

Equation 42 leads to:

$$
\begin{array}{r}
\max \left[a_{1}(x), a_{2}(x), \ldots, a_{n}(x)\right]<f_{p}(x)<\sqrt[p]{n} \max \left[a_{1}(x), a_{2}(x), \ldots, a_{n}(x)\right] \\
\forall p \in \mathbb{N} .
\end{array}
$$

Since $\lim _{p \rightarrow+\infty} \sqrt[p]{n}=1$, it can be deduced that $\lim _{p \rightarrow+\infty} f_{p}(x)=\max \left[a_{1}(x), a_{2}(x), \ldots, a_{n}(x)\right]$. Mathematically, this can be written as:

$\forall \epsilon>0 \exists \eta>0$ tq $x>\eta \Rightarrow\left|f_{p}(x)-\max \left[a_{1}(x), a_{2}(x), \ldots, a_{n}(x)\right]\right|<\epsilon$

Thus, if $p$ is large enough, criteria $\tilde{C}_{n}$ have nearly the same extrema as $C_{n}$.

\section{Optimizing a structure using the modal criteria}

\subsection{Analysis of the criteria}

In this section, the criteria developed in the previous sections are analyzed. In order to simplify the analysis, only one excitation point placed on a hollow part bounding the first cavity $\mathcal{F}_{1}$ is considered. 
Values of criteria $C_{n}$ may change with the excitation frequency because of coefficient $\lambda(\omega)$. For example, figures 4, 5, 6, 7, 9 and 10 show the values of some parameters as a function of the excitation frequency $f$. It can be seen that the strength of each criterion depends on the excitation frequency. For example, it is interesting to note that criterion $C P 22$ increases more strongly than the others with frequency $f$. Therefore, the vibrational path through plate $\mathcal{P}$ becomes very significant at higher frequencies.

Notice that criteria $C P 13$ and $C H 2$ show peaks which correspond to the global modes of the structure, because the modal matrices of the structure's hollow parts are involved in these criteria.

[Figure 4 about here.]

[Figure 5 about here.]

[Figure 6 about here.]

[Figure 7 about here.]

[Figure 8 about here.]

[Figure 9 about here.]

[Figure 10 about here.]

\subsection{Results for the modal parameters}

In this section, the modal parameters $\tilde{\mathbf{G}}_{H 1}^{k}, \tilde{\mathbf{G}}_{H 2}^{k}, \tilde{\mathbf{G}}_{P 21}^{k}, \tilde{\mathbf{G}}_{P 22}^{k}, \tilde{\mathbf{G}}_{P 23}^{k}, \tilde{\mathbf{G}}_{P 11}^{k}, \tilde{\mathbf{G}}_{P 13}^{k}$ and $\tilde{\mathbf{G}}_{P 14}^{k}$ are analyzed. This will allow each of the modes responsible for the values of criteria $C_{n}$ to be defined. The modal parameters must be weighted with the previously defined coefficients $\lambda(\omega)$, in order to take the excitation frequency into account. Two cases of excitation are presented, at $50 \mathrm{~Hz}$ and $300 \mathrm{~Hz}$.

\subsubsection{Path through the first cavity}

Considering the vibrational path through the first cavity and the plate $\mathcal{P}$, three modal parameters need to be analyzed. Figures 11, 12 and 13 show the values of modal parameters $\tilde{\mathbf{G}}_{P 21}^{k}, \tilde{\mathbf{G}}_{P 22}^{k}$ and $\tilde{\mathbf{G}}_{P 23}^{k}$ as a function of the mode number $k$, for an excitation frequency of $50 \mathrm{~Hz}$. Figures 14,15 and 16 show the same parameters for an excitation frequency of $300 \mathrm{~Hz}$.

[Figure 11 about here.]

[Figure 12 about here.]

[Figure 13 about here.]

[Figure 14 about here.]

[Figure 15 about here.] 
[Figure 16 about here.]

Figure 12 shows that only one mode of the plate $\mathcal{P}$ is responsible for the transmission of vibrations between the first and second cavities. The criterion associated with this figure is $C_{P 22}$, and is related to the influence of pressure within the first cavity on plate $\mathcal{P}$. Given that the action of an acoustic fluid on a structure is not very significant when compared with that of a structure on a fluid, the parameter values given in figure 12 are very small. For an influence to be exerted on the path through the first cavity, which is the object of this section, one could, for example, restrict the influence of the mode shown in figure 12

\subsubsection{Path through the hollow parts}

For the vibrational path through the hollow parts, two modal parameters have to be analyzed. Figures 17 and 18 show the values of modal parameters $\tilde{\mathbf{G}}_{H 1}^{k}$ and $\tilde{\mathbf{G}}_{H 2}^{k}$ as a function of the mode number $k$ for an excitation frequency of $50 \mathrm{~Hz}$. Figures 17 and 18 show the same parameters for an excitation frequency of $300 \mathrm{~Hz}$.

[Figure 17 about here.]

[Figure 18 about here.]

[Figure 19 about here.]

[Figure 20 about here.]

It can be seen in figures 17 and 18 that many of the modes are strong. The values of these parameters increase with increasing excitation frequency. However, the values for criterion $C_{H 2}$ are much smaller than the values for criterion $C_{H 1}$. It is then possible to choose to optimize the structure using $C_{H 1}$, which seems to have a stronger influence on the transmission of vibrations. Although the influence of criterion $\mathrm{CH} 2$ appears to be smaller, its use in the optimization of the structure allows the vibrational path through the plates to be studied, as described in the following section.

\subsubsection{Path through the hollow parts and the plates}

For the vibrational path through the hollow parts and the plates, three modal parameters should be analyzed. Figures 21 and 22 show the values of modal parameters $\tilde{\mathbf{G}}_{P 11}^{k}$ and $\tilde{\mathbf{G}}_{P 13}^{k}$ as a function of mode number $k$ for an excitation frequency of $50 \mathrm{~Hz}$. It should be noted that $\tilde{\mathbf{G}}_{P 14}^{k}=\tilde{\mathbf{G}}_{H 2}^{k}$, as plotted in figure 18 . Figures 23 and 24 show the values of modal parameters $\tilde{\mathbf{G}}_{P 11}^{k}$ and $\tilde{\mathbf{G}}_{P 13}^{k}$ as a function of the mode number $k$ for an excitation frequency of $300 \mathrm{~Hz}$.

[Figure 21 about here.]

[Figure 22 about here.]

[Figure 23 about here.] 
[Figure 24 about here.]

As shown if figures 21 and 22 many modes are strong. Just as in the previous section, it is then possible to optimize the structure using criterion $\mathbf{C}_{P 14}=\mathbf{C}_{H 2}^{k}$, so as to minimize the transmission of vibrations through two different paths. It is also possible to minimize criterion $C_{P 11}$, which is larger than $C_{P 13}$.

\section{Results and validation on a simple example}

Since many criteria can be found, it is very difficult to show how efficient the method is. This is the reason for which it was decided to validate the derived criteria with a simple example.

The system shown in figure 25 is considered, in which a cavity is bounded by rigid walls and a single plate.

[Figure 25 about here.]

For such a system, there are only two criteria, which can be expressed as follows:

$$
\begin{aligned}
& C_{D}=\frac{\boldsymbol{\Phi}_{f}^{k}}{-\omega^{2} \boldsymbol{m}_{f}+\boldsymbol{k}_{f}}\left(\omega^{2} \mathbf{M}_{F E}^{k}+\mathbf{M}_{F S}^{k} \tilde{\boldsymbol{\Phi}}_{s} \boldsymbol{\Psi}_{s}\right) \\
& C_{P}=\frac{\boldsymbol{\Phi}_{f}^{k}}{-\omega^{2} \boldsymbol{m}_{f}+\boldsymbol{k}_{f}}\left(-\mathbf{M}_{F S}^{k} \tilde{\boldsymbol{\Phi}}_{s}\right)
\end{aligned}
$$

where $\boldsymbol{\Phi}_{f}$ is the matrix of the acoustic modes, $\boldsymbol{\Phi}_{s}$ is the matrix of the modes of the plate, and $\boldsymbol{\Psi}_{s}$ is the matrix of the static modes:

$$
\begin{aligned}
& \mathbf{u}_{f}=\boldsymbol{\Phi}_{f} \mathbf{q}_{f} \\
& \mathbf{u}_{s}=\boldsymbol{\Phi}_{s} \mathbf{q}_{s}+\boldsymbol{\Psi}_{s} \mathbf{u}_{E}
\end{aligned}
$$

In order to validate our methodology for the determination of suitable criteria, it is proposed to compute the latter for two values of plate thickness. Since plate thickness does not change criterion $C_{D}$, which is directly related to the excitation point $\mathbf{u}_{E}$, only one criterion remains. It is then possible to study the variation of this criterion and the pressure level within the cavity.

Figures 26, 27 and 28 show how the criteria change as a function of the pressure level within the cavity for two different thickness values. The pressure level has been normalized with respect to its value for the initial thickness.

[Figure 26 about here.]

[Figure 27 about here.]

[Figure 28 about here.]

These figures show that criterion $C_{P}$ decreases as a function of pressure level. Criterion $C_{D}$ remains constant because the plate thickness is not taken into account in this criterion. This simple example shows that the criteria proposed in the previous sections can be very useful. 


\section{Conclusion}

In the present paper several criteria, corresponding to different vibrational propagation paths, and based on modal analysis of a coupled system, have been proposed. These illustrate the relative influence of each mode on noise propagation, and show which part of the system has the strongest influence on the generation of noise inside the structure.

Initially, a complex system was considered in order to provide an overview of the various criteria which may exist. A simple example was then introduced in order to validate the efficiency of the method. Although the criteria were found by assuming a specific structure, it is possible to apply the same method to any vibroacoustic system.

The goal of this paper was to derive criteria which can allow a vibroacoustic system to be optimized. The optimization of such a system lies beyond the scope of the present paper.

\section{References}

[1] P. Lemerle, Optimisation des structures selon des critères imposés par la discrétion acoustique des navires, Thèse de doctorat, École Centrale de Lyon (1994).

[2] L. Jézéquel, H. D. Setio, Component modal synthesis methods based on hybrid models, part i : Theory of hybrid models and modal truncation methods, ASME Journal of Applied Mechanics 61 (1994) 100-108.

[3] L. Jézéquel, H. D. Setio, Component modal synthesis methods based on hybrid models, part ii : Numerical tests and experimental identification of hybrid model, ASME Journal of Applied Mechanics 61 (1994) 109-116.

[4] S. Besset, L. Jézéquel, Modal analysis of hollow parts of a structure, in: IMAC XXIV: A Conference \& Exposition on Structural Dynamics, 2005.

[5] S. Besset, L. Jézéquel, Optimization of structural dynamic behaviour based on effective modal parameters, International journal for numerical methods in engineering Accepted on August 27, 2006.

[6] R. Ohayon, Reduced models for fluid-structure interaction problems, International Journal for Numerical Methods in Engineering 60 (2003) 139-152.

[7] R. Ohayon, Reduced symetric models for modal analysis of internal structuralacoustic and hydroelastic-sloshing systems, Computer Methods in Applied Mechanics and Engineering 190 (2001) 3009-3019.

[8] G. E. Sandberg, P.-A. Hansson, M. Gustavsson, Domain decomposition in acoustic and structure-acoustic analysis, Computer Methods in Applied Mechanics and Engineering 190 (2001) 2979-2988.

[9] R. R. Craig, M. C. C. Bampton, Coupling of substructures for dynamic analysis, AIAA Journal 6 (1968) 1313-1321. 
[10] C. Farhat, M. Géradin, On the general solution by a direct method on a largescale singular system of linear equations: Application to the analysis of floating structures, International journal for numerical methods in engineering 41 (1998) 675-696. 


\section{List of Figures}

$1 \quad$ Structure to optimize . . . . . . . . . . . . . . . . . . . 19

2 The hollow part to analyze . . . . . . . . . . . . . . 20

3 An element of the hollow part ................ . . 21

4 Values of parameter $\mathrm{CH} 1$ as a function of $f$. . . . . . . . . . 22

5 Values of parameter $\mathrm{CH} 2$ as a function of $f \ldots \ldots 23$

6 Values of parameter $C P 21$ as a function of $f \ldots \ldots 24$

$7 \quad$ Values of parameter $C P 22$ as a function of $f \ldots \ldots$. . . . . . 25

$8 \quad$ Values of parameter $C P 23$ as a function of $f \ldots \ldots$. . . . . . . . 26

9 Values of parameter $C P 11$ as a function of $f \ldots \ldots 27$

10 Values of parameter $C P 13$ as a function of $f \ldots \ldots$

\begin{tabular}{|l|l|l|l|l|l}
\hline $11 \quad$ Values of parameter & $\lambda \tilde{\mathbf{G}}_{P 21}^{k}$ & as a function of $k-50 \mathrm{~Hz}$ & 29
\end{tabular}

\begin{tabular}{|l|l|l|l|l|l}
\hline 12 & Values of parameter & $\lambda \tilde{\mathbf{G}}_{p 22}^{k}$ & as a function of $k-50 \mathrm{~Hz}$ & $\ldots$ & 30
\end{tabular}

\begin{tabular}{|l|l|l|l|l}
\hline $13 \quad$ Values of parameter & $\lambda \tilde{\mathbf{G}}_{P 23}^{k}$ & as a function of $k-50 \mathrm{~Hz}$ & $\ldots$ & $\ldots$
\end{tabular}

\begin{tabular}{|l|l|l|l|l}
\hline $14 \quad$ Values of parameter & $\lambda \tilde{\mathbf{G}}_{P 21}^{k}$ & as a function of $k-300 \mathrm{~Hz}$
\end{tabular}$\ldots . . . . \quad 32$

\begin{tabular}{|l|l|l|l|l}
\hline $15 \quad$ Values of parameter & $\lambda \tilde{\mathbf{G}}_{P 22}^{k}$ & as a function of $k-300 \mathrm{~Hz}$
\end{tabular}$\ldots . . . \quad 33$

\begin{tabular}{|l|l|l|l|l}
\hline $16 \quad$ Values of parameter & $\lambda \tilde{\mathbf{G}}_{P 23}^{k}$ & as a function of $k-300 \mathrm{~Hz}$
\end{tabular}$\ldots . . . \quad 34$

\begin{tabular}{|ll|l|l|l|l}
\hline $17 \quad$ Values of parameter & $\lambda \tilde{\mathbf{G}}_{H 1}^{k}$ & as a function of $k-50 \mathrm{~Hz}$
\end{tabular}$\ldots . . . .35$

\begin{tabular}{|l|l|l|l|l}
\hline 18 Values of parameter & $\lambda \tilde{\mathbf{G}}_{H 2}^{k}$ & as a function of $k-50 \mathrm{~Hz}$
\end{tabular}$\ldots . . . .36$

\begin{tabular}{|l|l|l|l}
\hline 19 Values of parameter & $\lambda \tilde{\mathbf{G}}_{H 1}^{k}$ & as a function of $k-300 \mathrm{~Hz}$
\end{tabular}$\ldots . . . \quad 37$

\begin{tabular}{|ll|l|l|l|l}
\hline 20 & Values of parameter & $\lambda \tilde{\mathbf{G}}_{H 2}^{k}$ & as a function of $k-300 \mathrm{~Hz}$ & $\ldots$ &
\end{tabular}

\begin{tabular}{|ll|l|l|l}
21 & Values of parameter & $\lambda \tilde{\mathbf{G}}_{P 11}^{k}$ & as a function of $k-50 \mathrm{~Hz}$ & $\ldots$
\end{tabular}

\begin{tabular}{|l|l|l|l}
\hline 22 & Values of parameter & $\lambda \tilde{\mathbf{G}}_{P 13}^{k}$ & as a function of $k-50 \mathrm{~Hz}$
\end{tabular}$\ldots . . .40$

\begin{tabular}{|l|l|l|l|l}
\hline 23 & Values of parameter & $\lambda \tilde{\mathbf{G}}_{P 11}^{k}$ & as a function of $k-300 \mathrm{~Hz}$
\end{tabular}$\ldots . . . .41$

\begin{tabular}{|l|l|l|l|l}
\hline $24 \quad$ Values of parameter & $\lambda \tilde{\mathbf{G}}_{P 13}^{k}$ & as a function of $k-300 \mathrm{~Hz}$
\end{tabular}$\ldots . . . .42$

25 System used for validation . . . . . . . . . . . . . . . . . . 43

26 Values of the criteria, $f=200 H z \ldots \ldots$. . . . . . . . . . 44

27 Values of the criteria, $f=300 \mathrm{~Hz} \ldots \ldots . \ldots . \ldots 45$

28 Values of the criteria, $f=400 H z$. . . . . . . . . . 46 


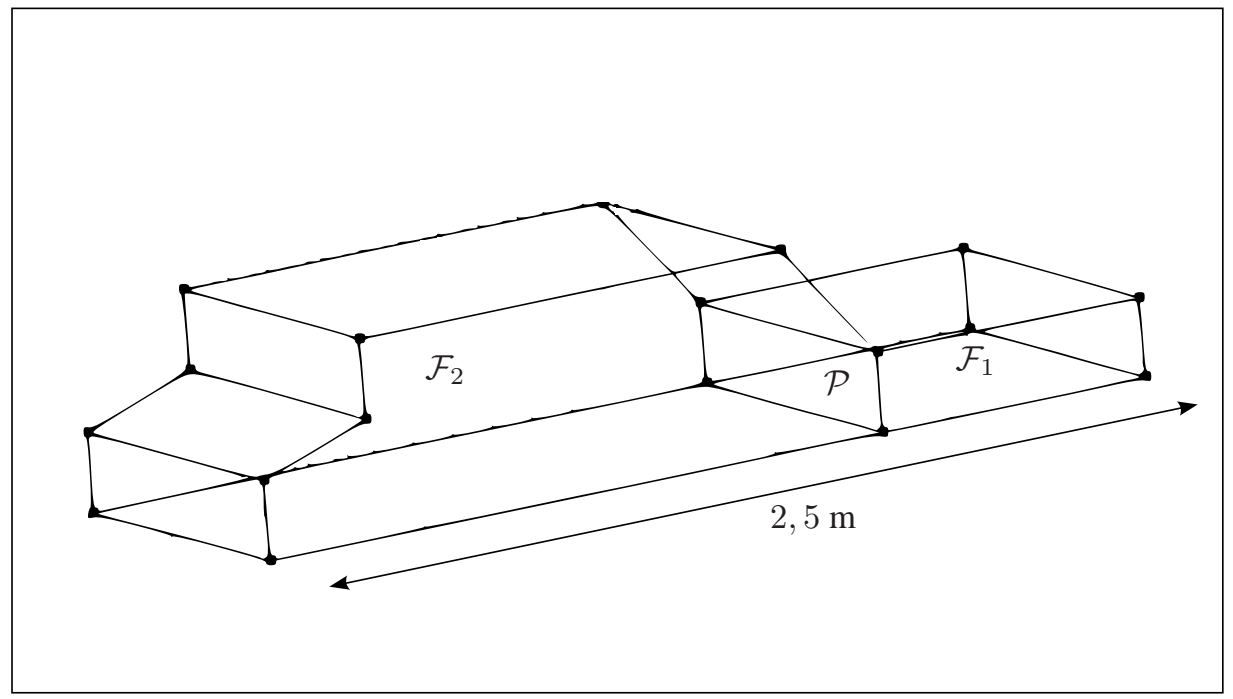

Figure 1: Structure to optimize 


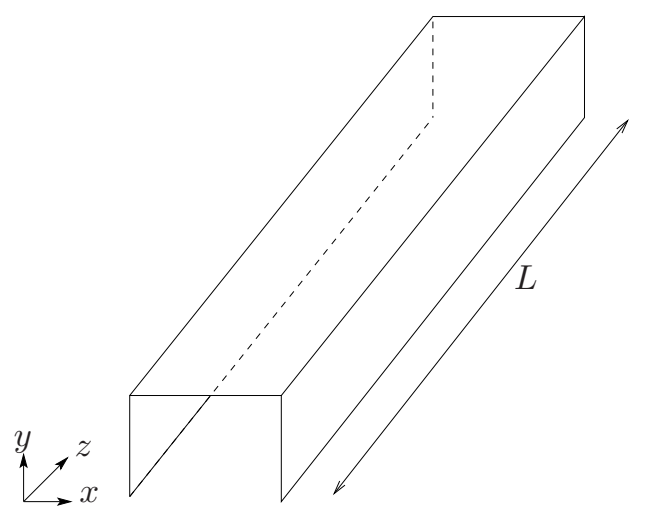

Figure 2: The hollow part to analyze 


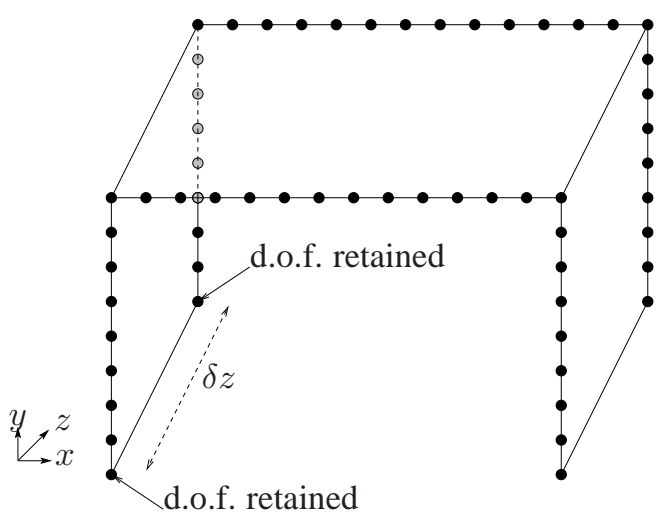

Figure 3: An element of the hollow part 


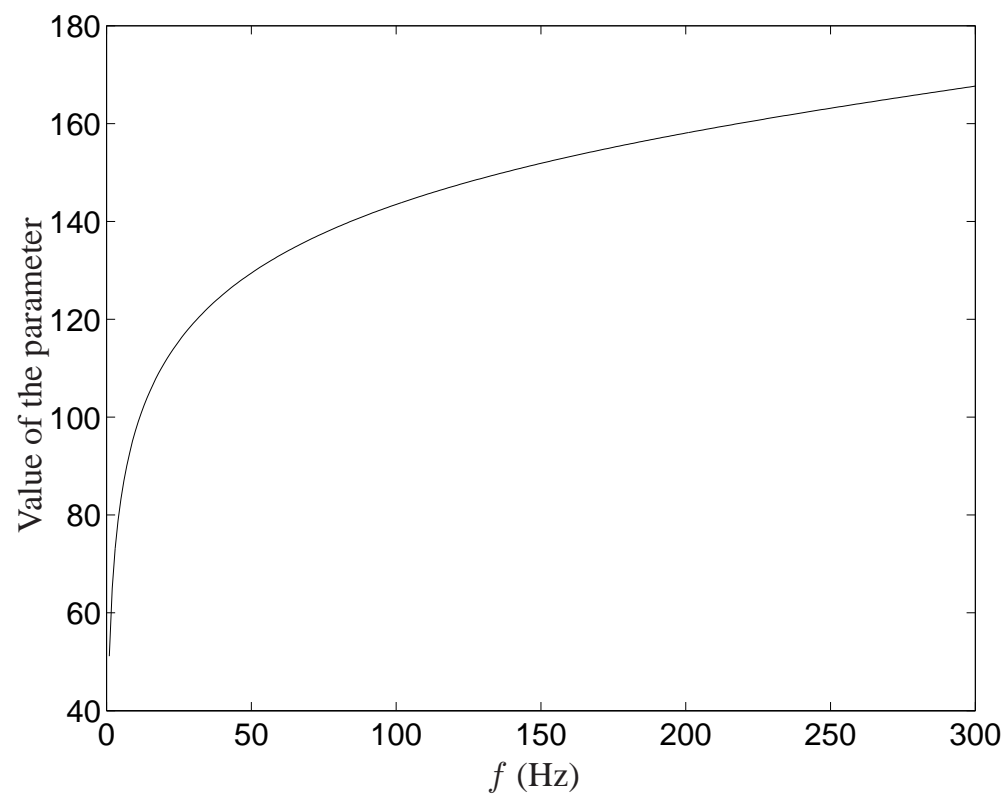

Figure 4: Values of parameter $C H 1$ as a function of $f$ 


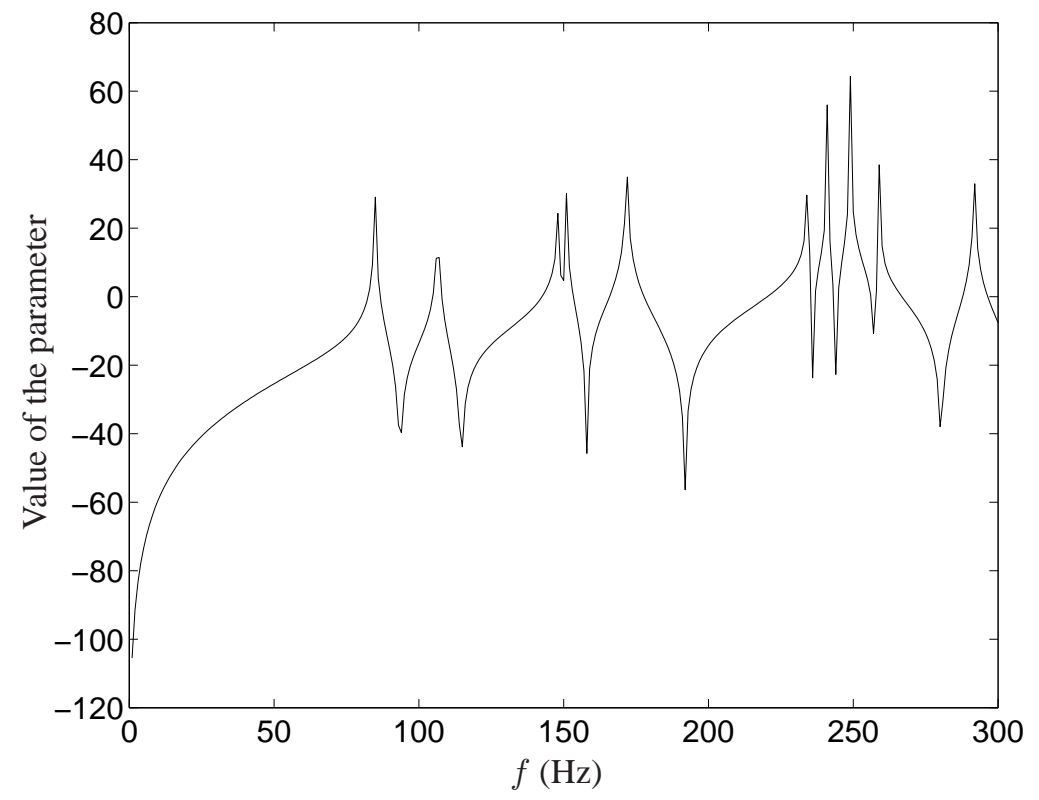

Figure 5: Values of parameter $\mathrm{CH} 2$ as a function of $f$ 


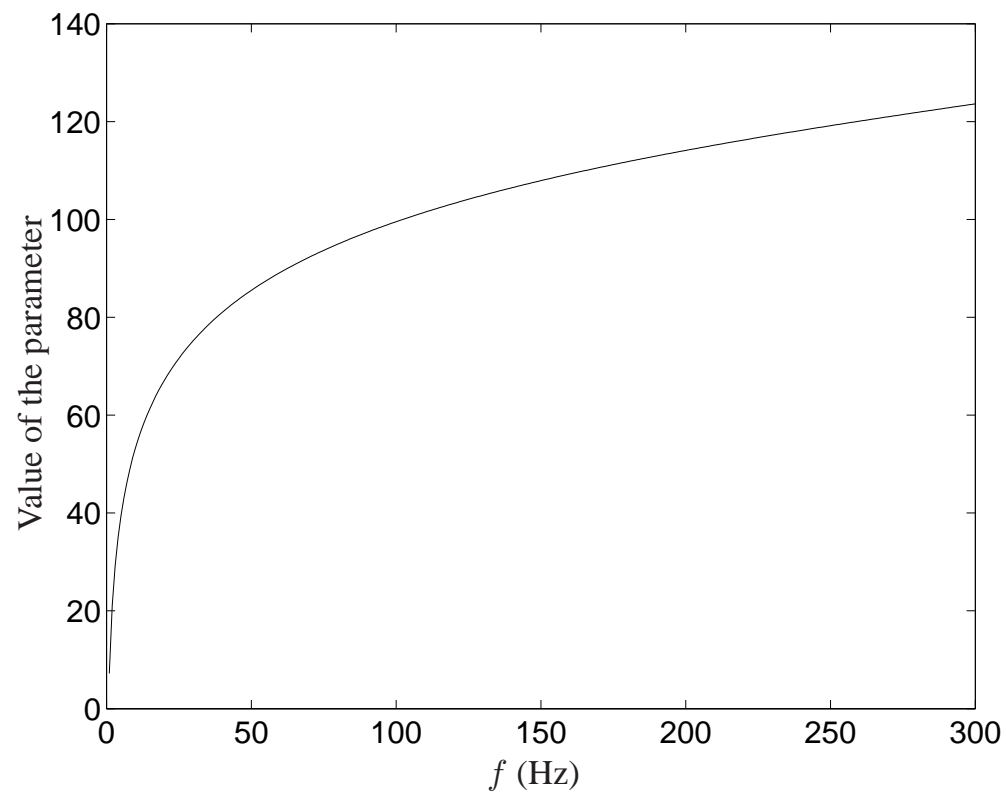

Figure 6: Values of parameter CP21 as a function of $f$ 


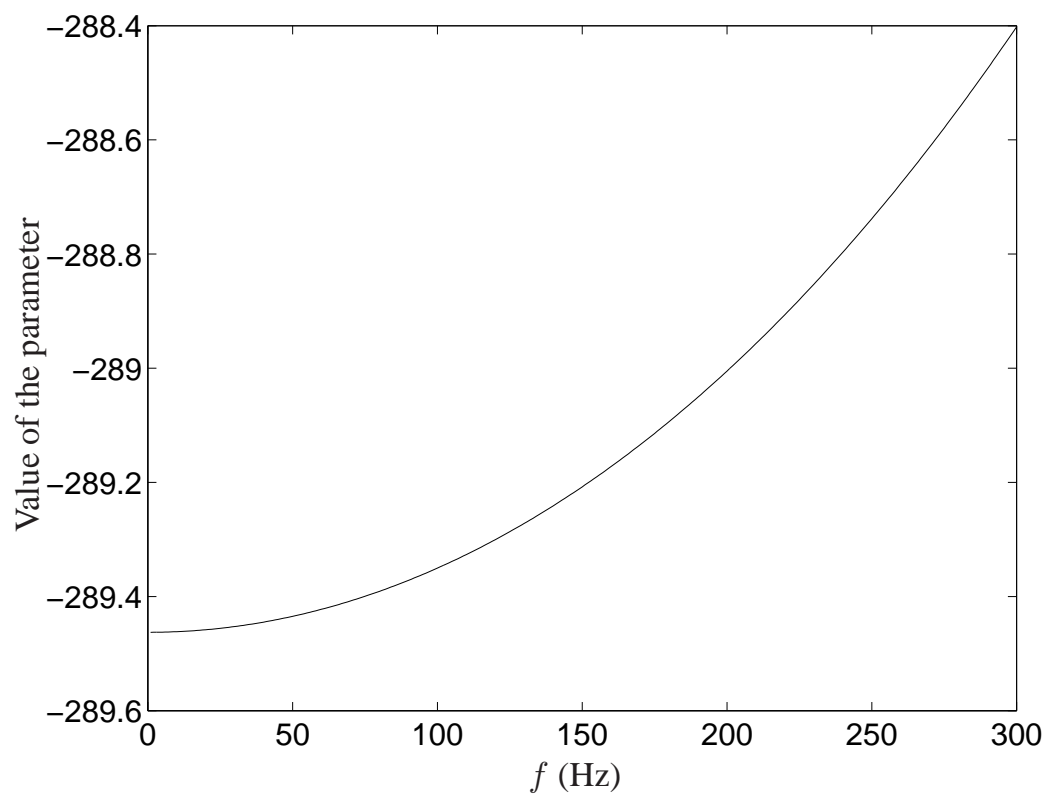

Figure 7: Values of parameter CP22 as a function of $f$ 


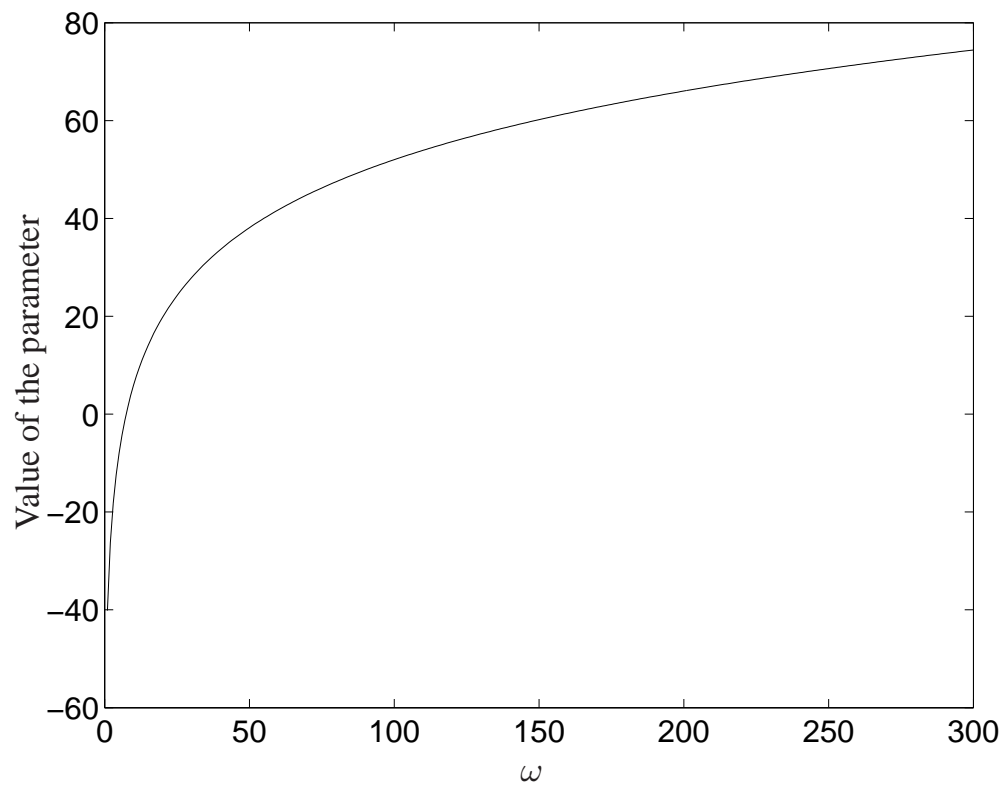

Figure 8: Values of parameter CP23 as a function of $f$ 


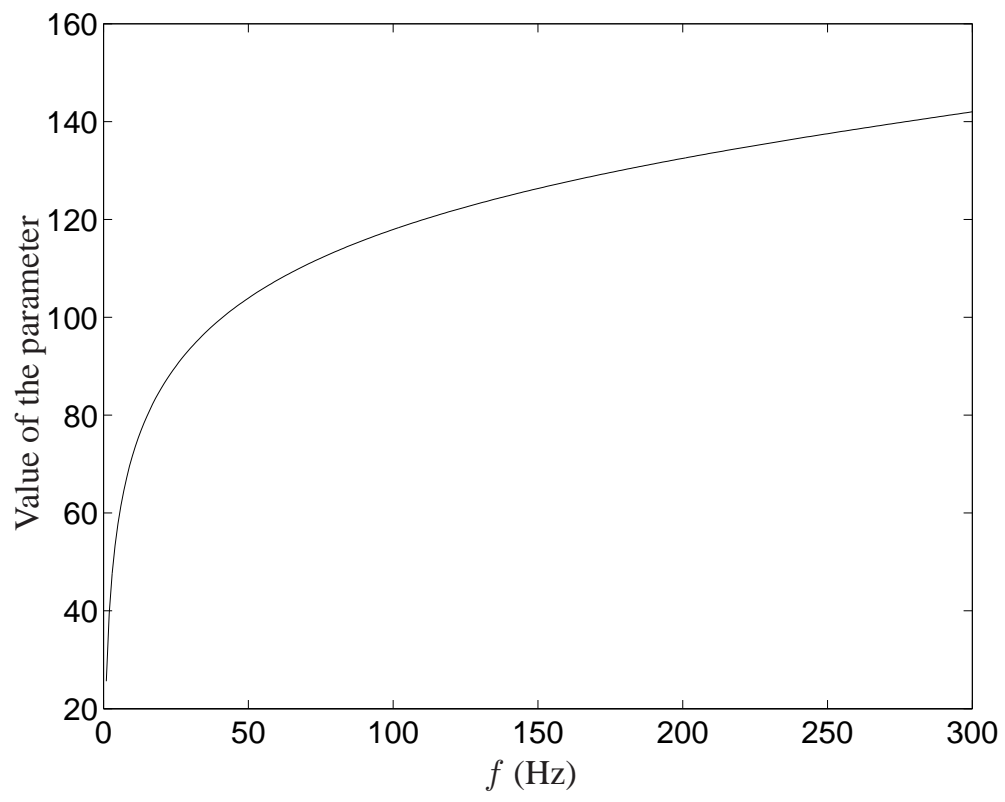

Figure 9: Values of parameter $C P 11$ as a function of $f$ 


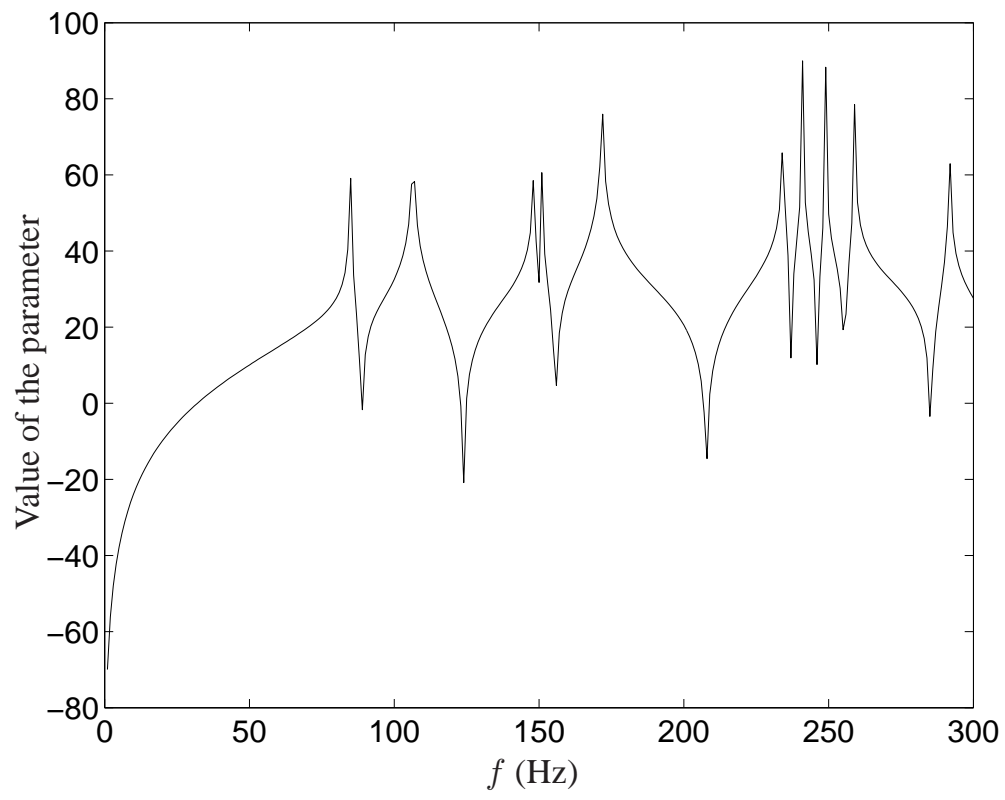

Figure 10: Values of parameter $C P 13$ as a function of $f$ 


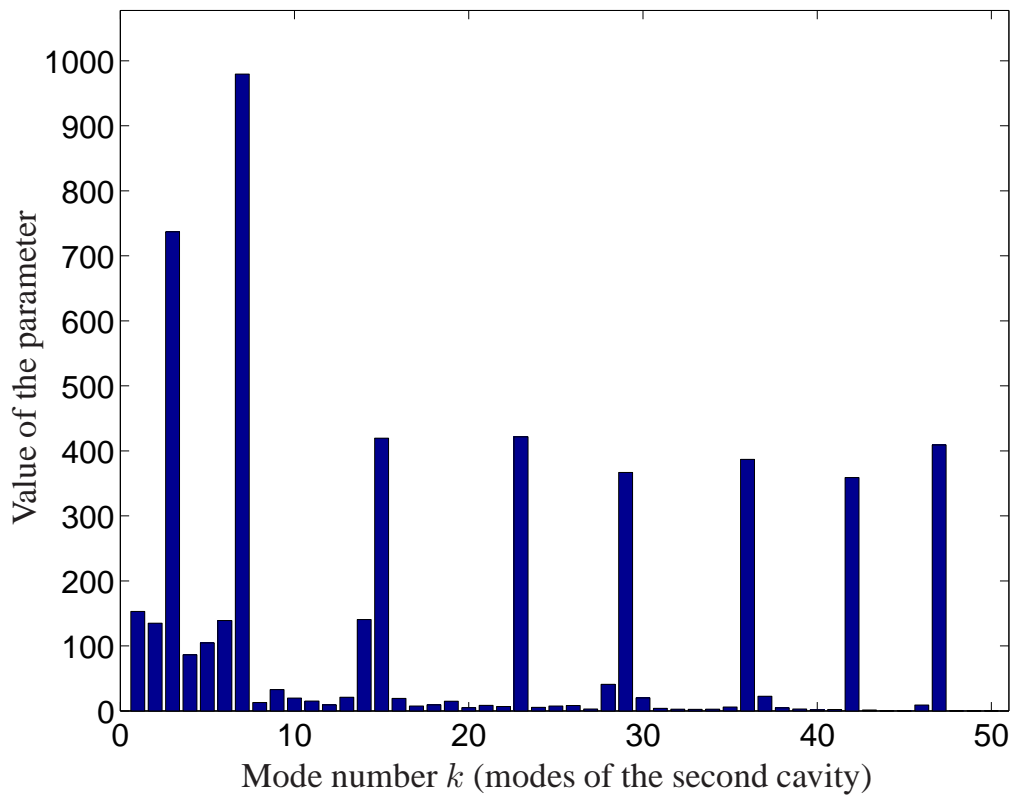

Figure 11: Values of parameter $\left|\lambda \tilde{\mathbf{G}}_{P 21}^{k}\right|$ as a function of $k-50 \mathrm{~Hz}$ 


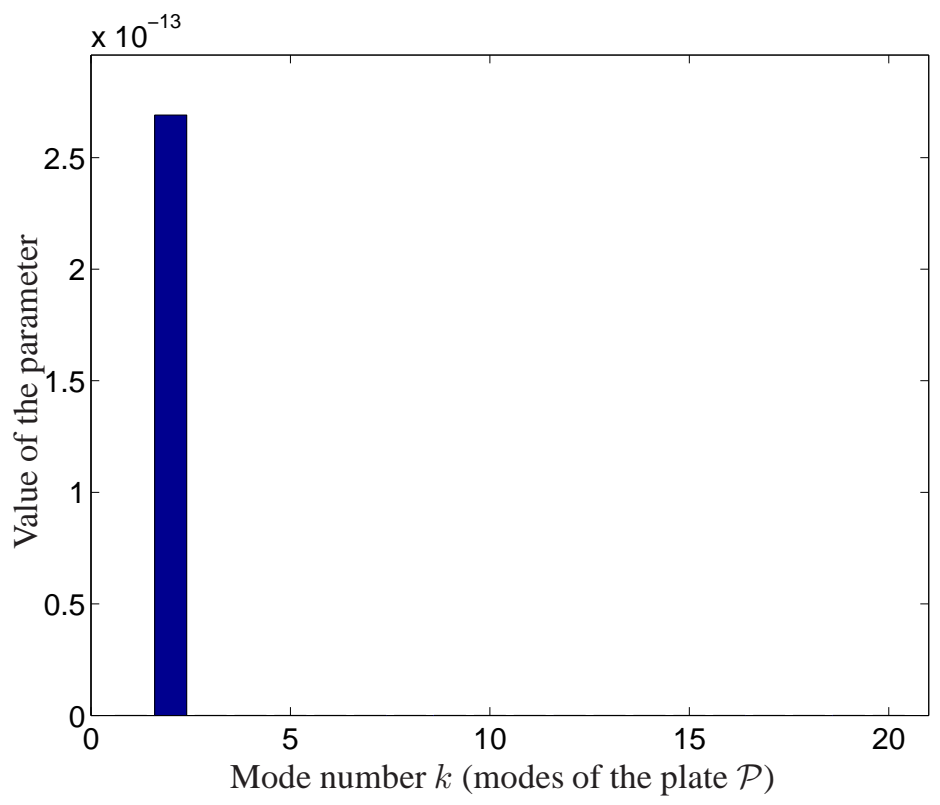

Figure 12: Values of parameter $\left|\lambda \tilde{\mathbf{G}}_{P 22}^{k}\right|$ as a function of $k-50 \mathrm{~Hz}$ 


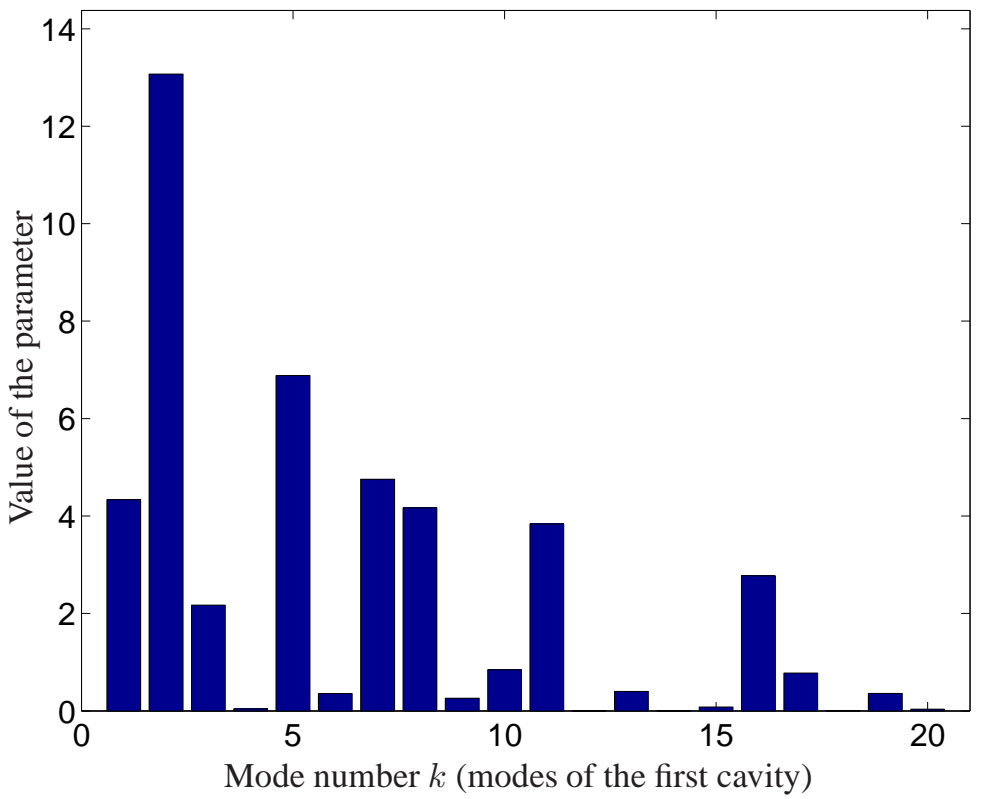

Figure 13: Values of parameter $\left|\lambda \tilde{\mathbf{G}}_{P 23}^{k}\right|$ as a function of $k-50 \mathrm{~Hz}$ 


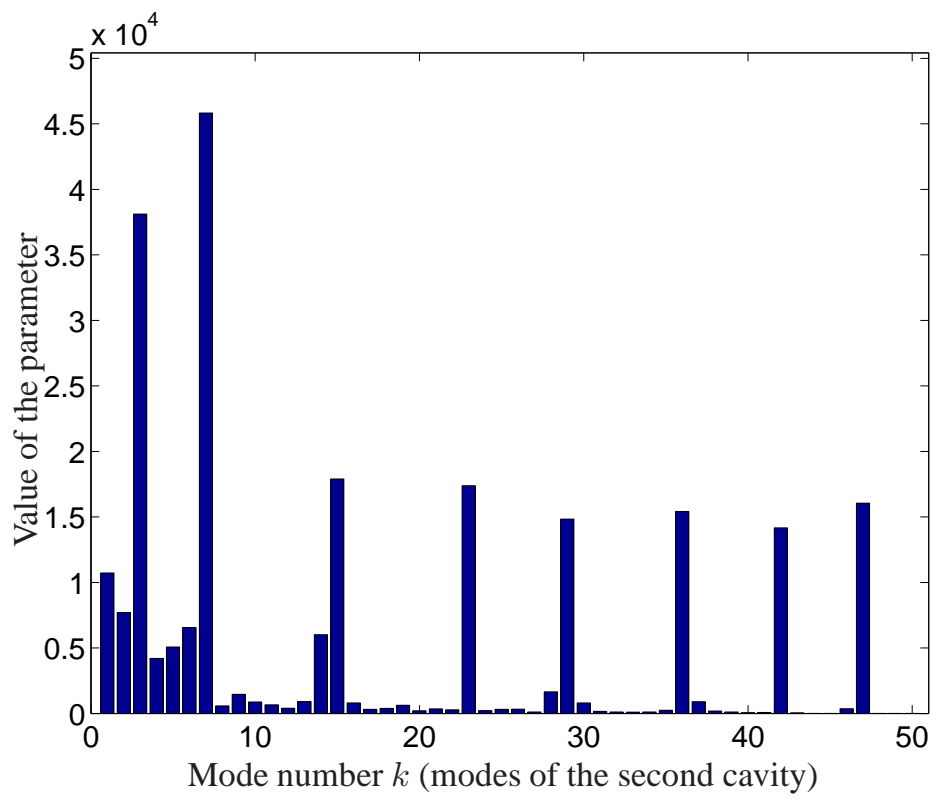

Figure 14: Values of parameter $\left|\lambda \tilde{\mathbf{G}}_{P 21}^{k}\right|$ as a function of $k-300 \mathrm{~Hz}$ 


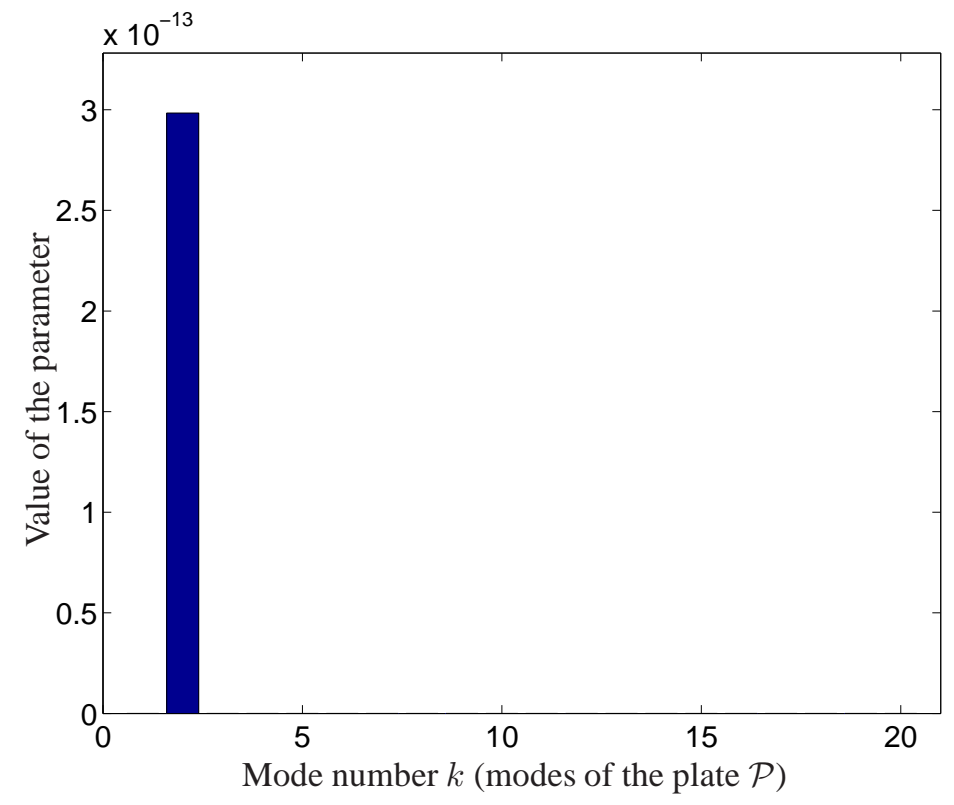

Figure 15: Values of parameter $\left|\lambda \tilde{\mathbf{G}}_{P 22}^{k}\right|$ as a function of $k-300 \mathrm{~Hz}$ 


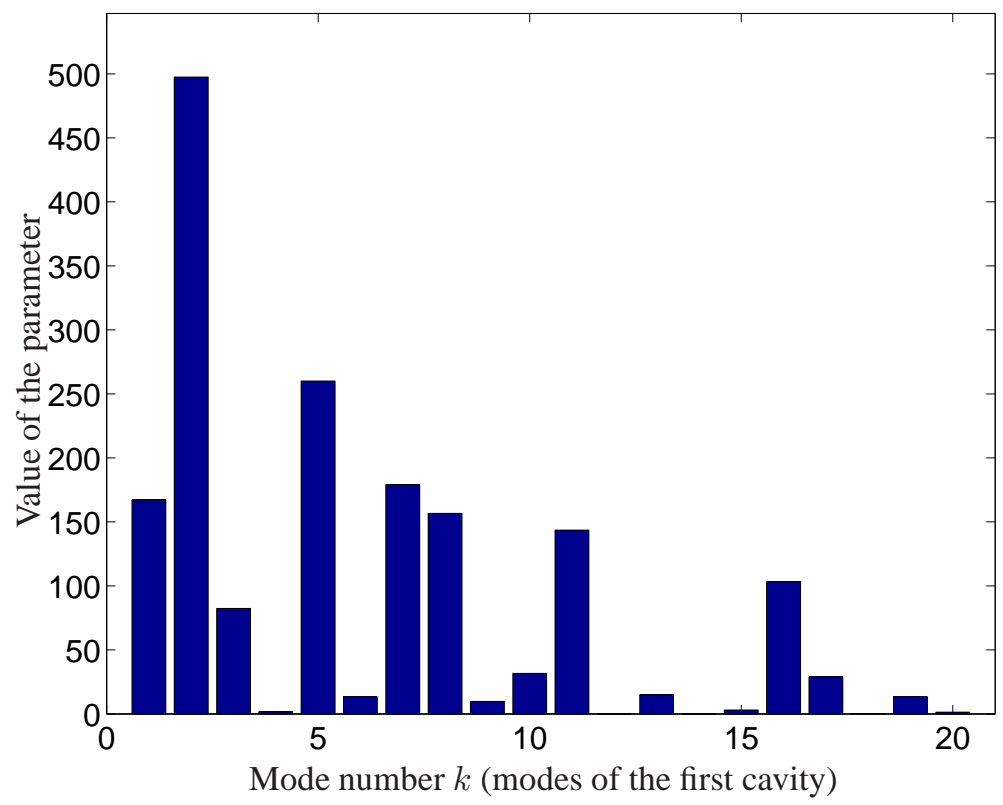

Figure 16: Values of parameter $\left|\lambda \tilde{\mathbf{G}}_{P 23}^{k}\right|$ as a function of $k-300 \mathrm{~Hz}$ 


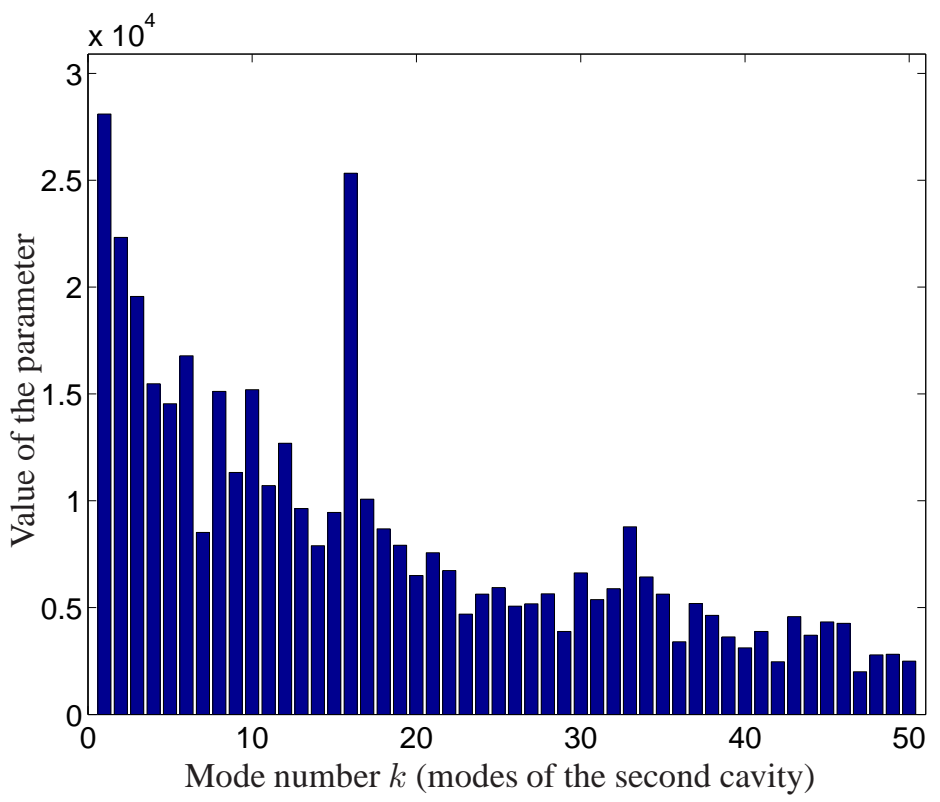

Figure 17: Values of parameter $\left|\lambda \tilde{\mathbf{G}}_{H 1}^{k}\right|$ as a function of $k-50 \mathrm{~Hz}$ 


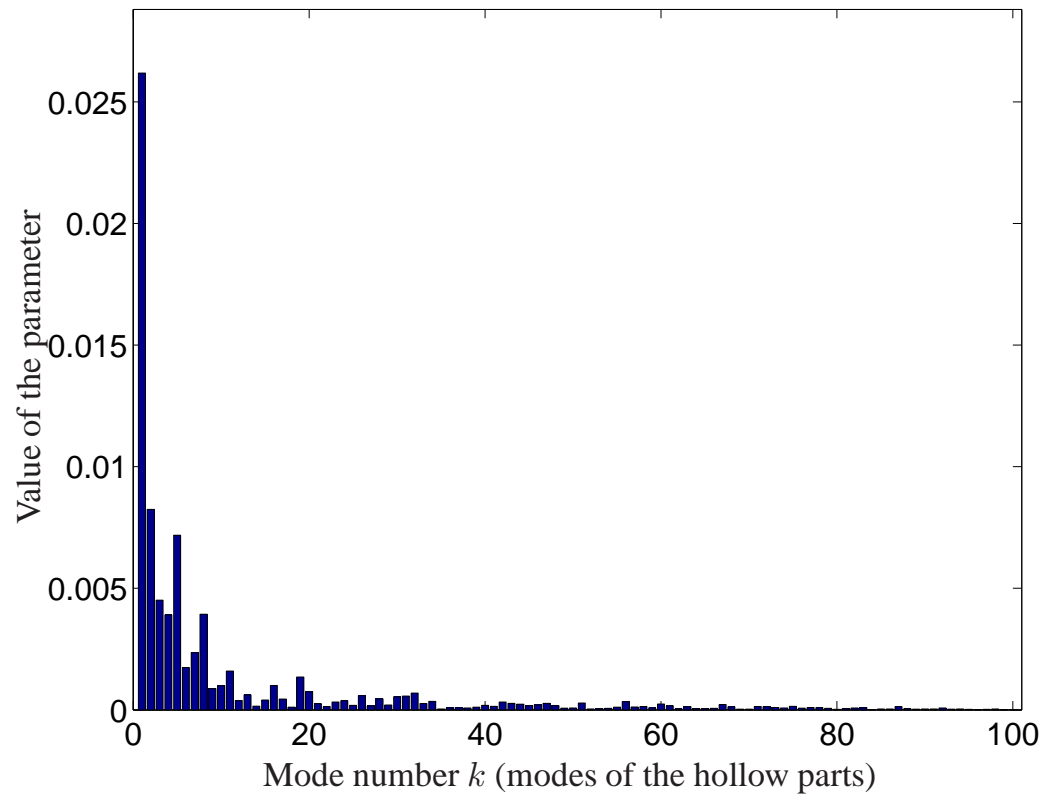

Figure 18: Values of parameter $\left|\lambda \tilde{\mathbf{G}}_{H 2}^{k}\right|$ as a function of $k-50 \mathrm{~Hz}$ 


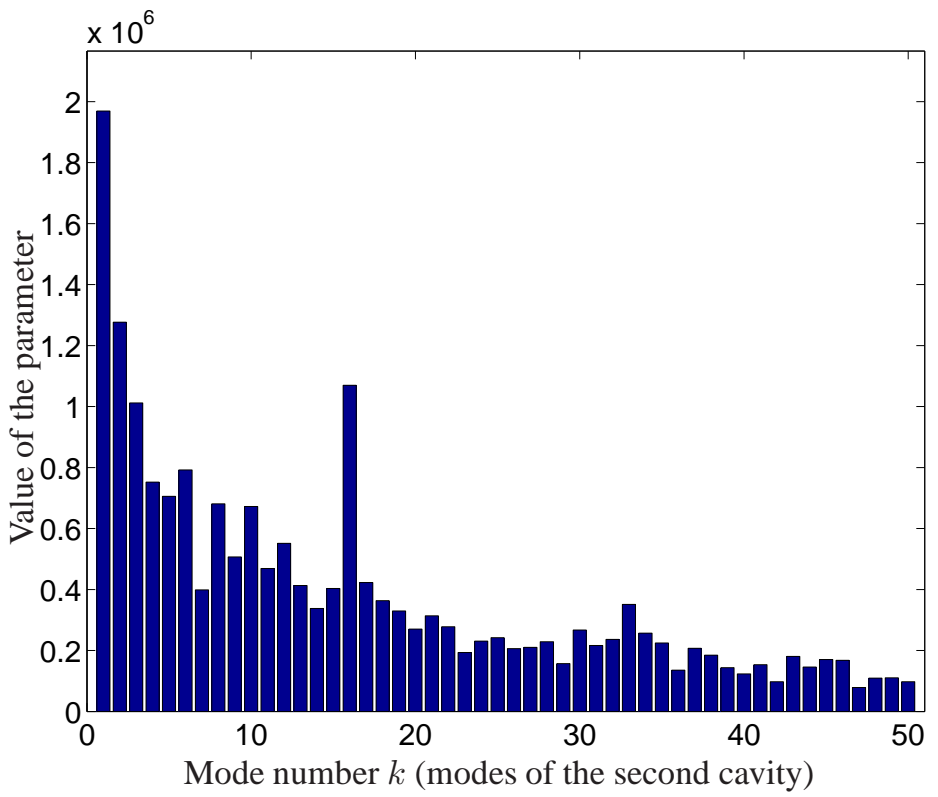

Figure 19: Values of parameter $\left|\lambda \tilde{\mathbf{G}}_{H 1}^{k}\right|$ as a function of $k-300 \mathrm{~Hz}$ 


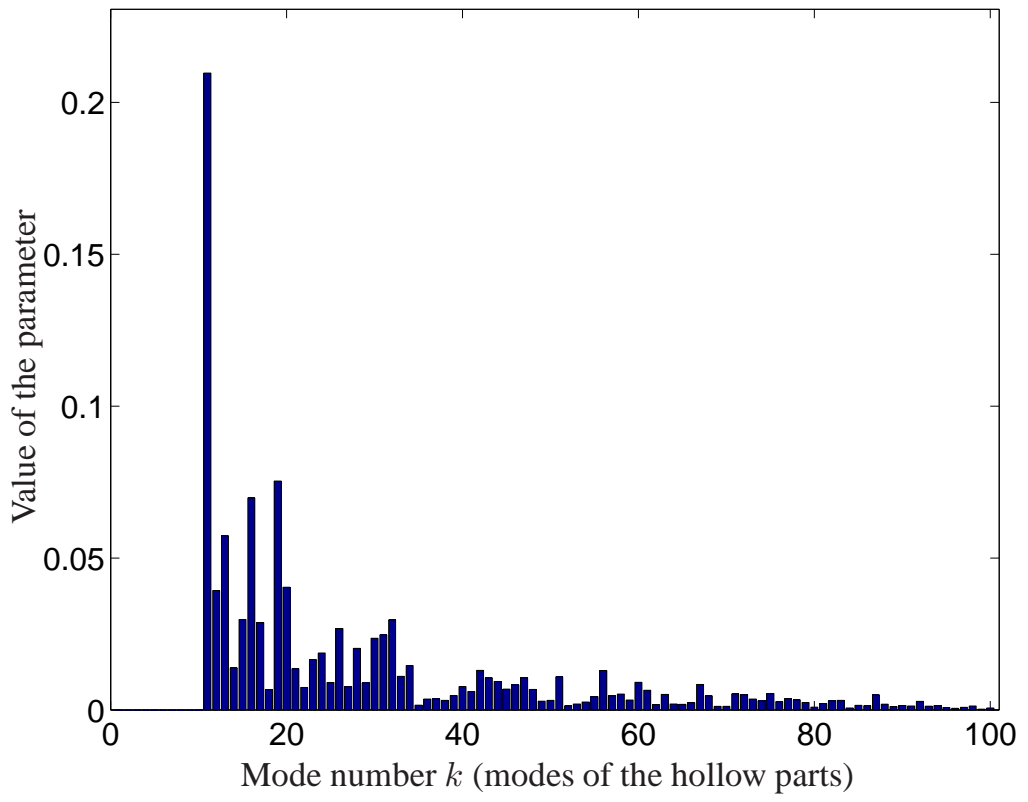

Figure 20: Values of parameter $\left|\lambda \tilde{\mathbf{G}}_{\mathrm{H} 2}^{k}\right|$ as a function of $k-300 \mathrm{~Hz}$ 


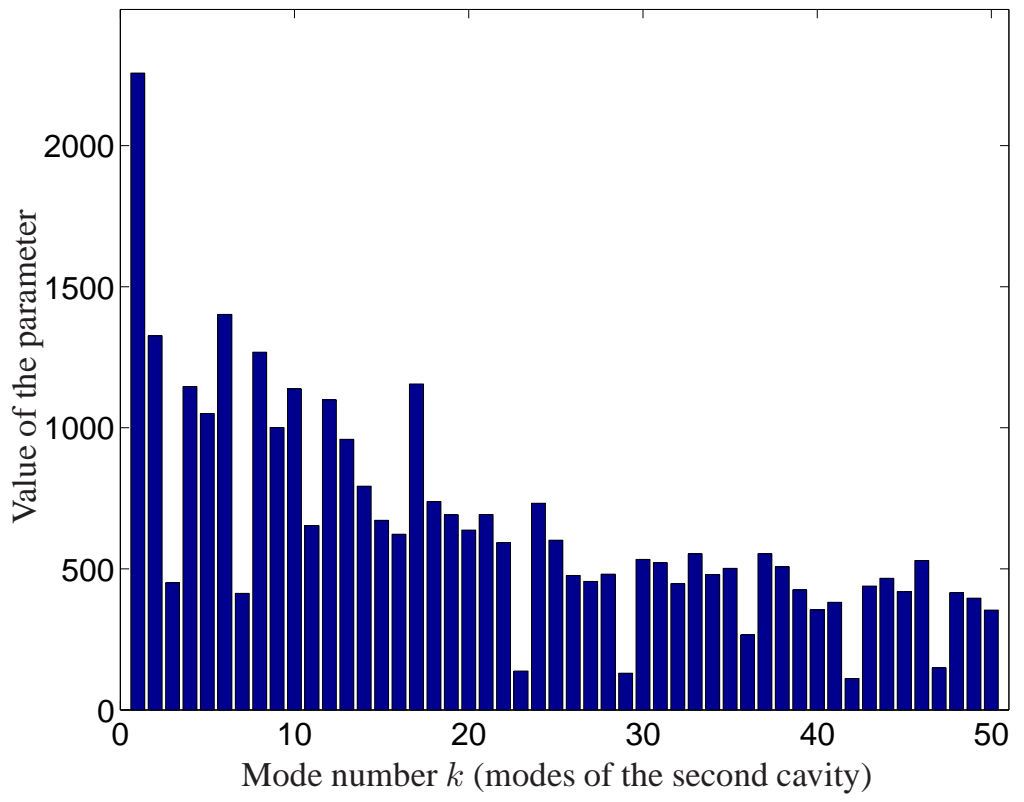

Figure 21: Values of parameter $\left|\lambda \tilde{\mathbf{G}}_{P 11}^{k}\right|$ as a function of $k-50 \mathrm{~Hz}$ 


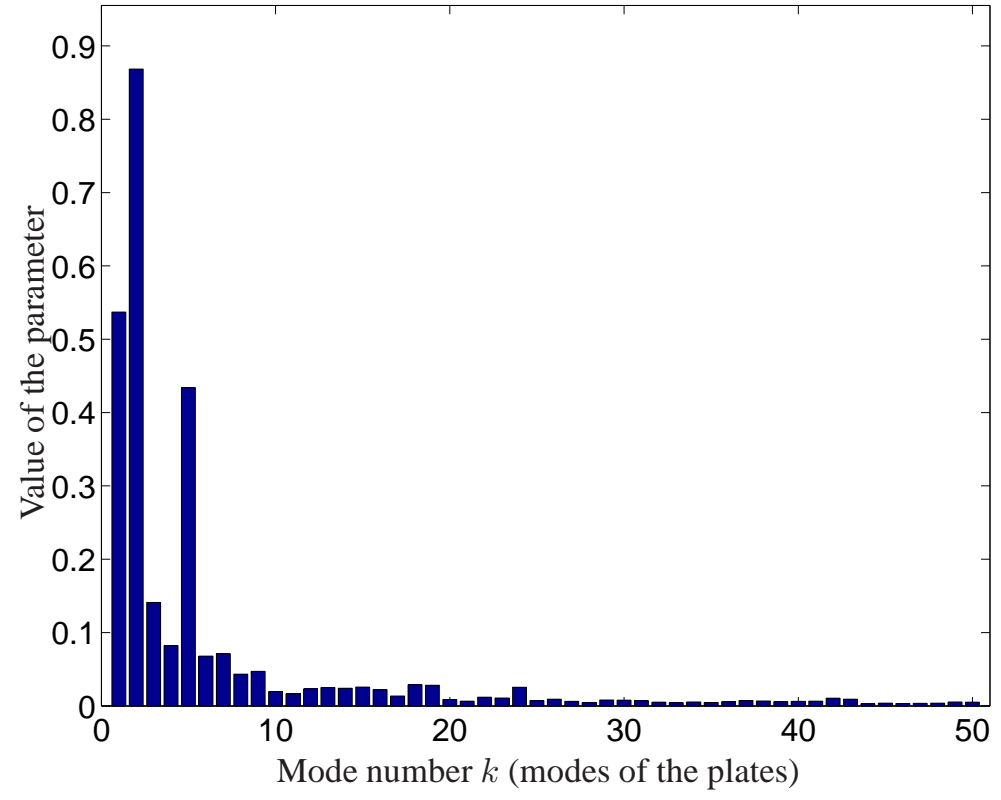

Figure 22: Values of parameter $\left|\lambda \tilde{\mathbf{G}}_{P 13}^{k}\right|$ as a function of $k-50 \mathrm{~Hz}$ 


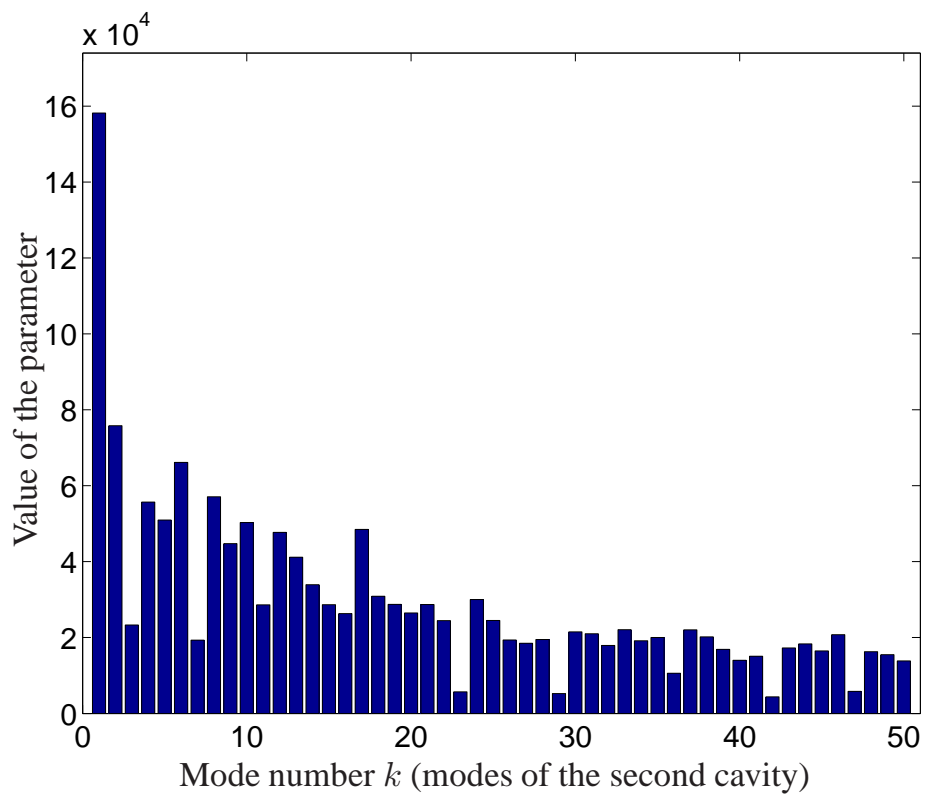

Figure 23: Values of parameter $\left|\lambda \tilde{\mathbf{G}}_{P 11}^{k}\right|$ as a function of $k-300 \mathrm{~Hz}$ 


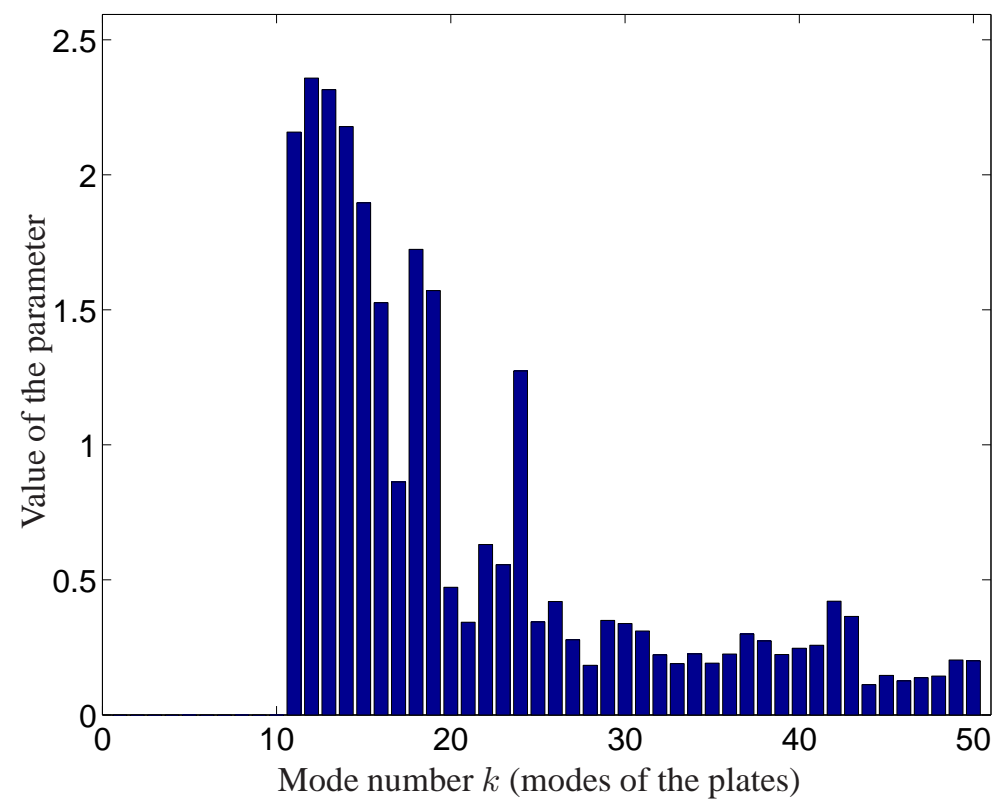

Figure 24: Values of parameter $\left|\lambda \tilde{\mathbf{G}}_{P 13}^{k}\right|$ as a function of $k-300 \mathrm{~Hz}$ 


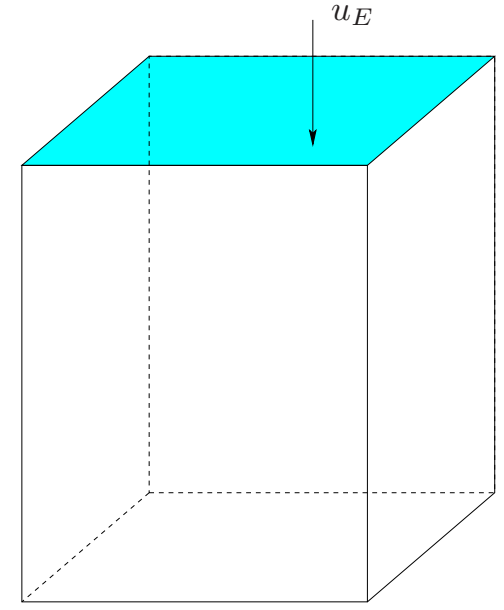

Figure 25: System used for validation 

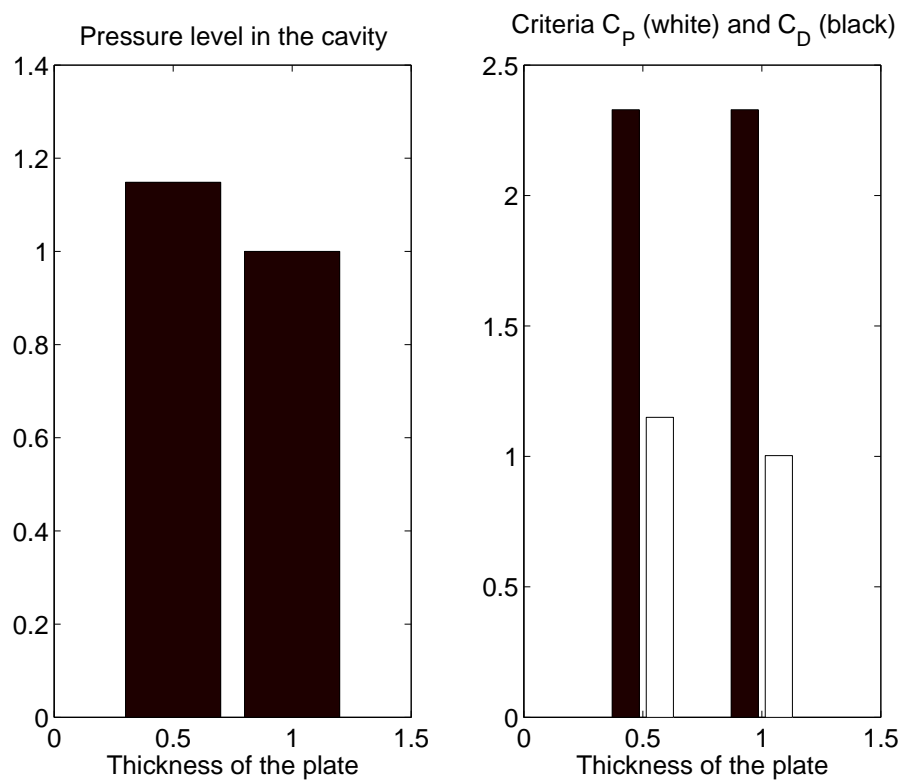

Figure 26: Values of the criteria, $f=200 \mathrm{~Hz}$ 

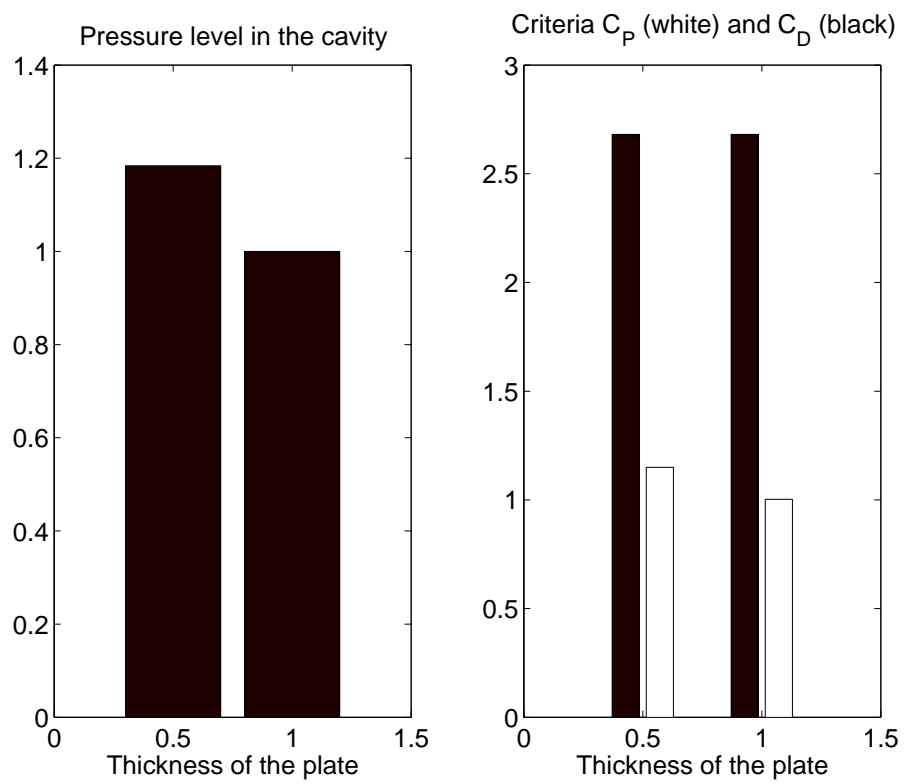

Figure 27: Values of the criteria, $f=300 \mathrm{~Hz}$ 

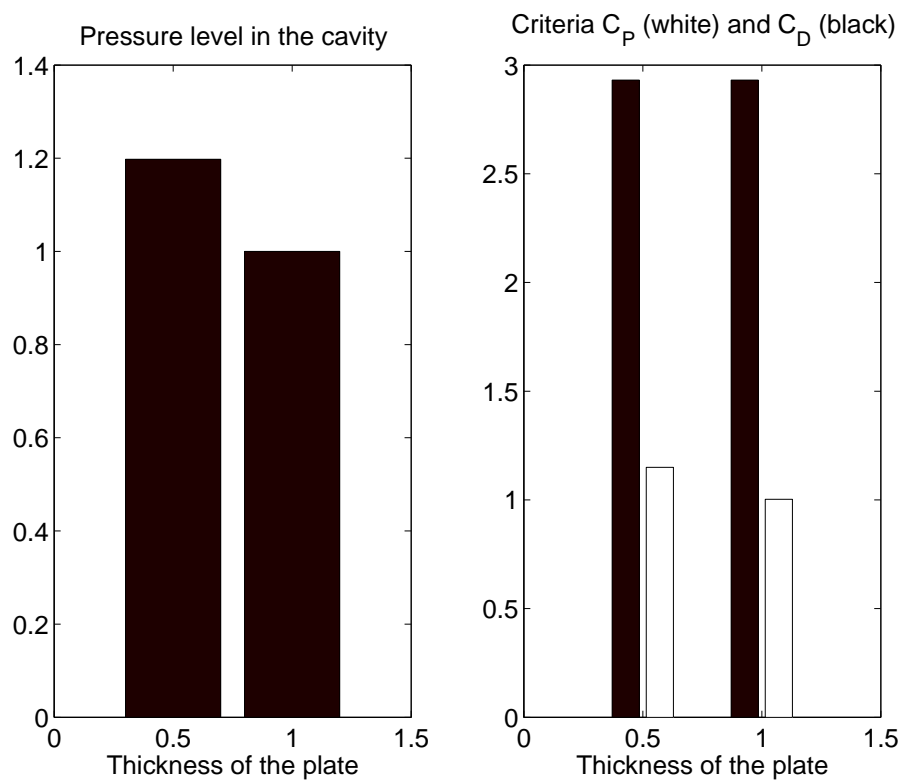

Figure 28: Values of the criteria, $f=400 \mathrm{~Hz}$ 